\title{
Detecting changes in dynamical structures in synchronous neural oscillations using probabilistic inference
}

\author{
Hiroshi Yokoyama ${ }^{a, b, *}$, Keiichi Kitajo ${ }^{a, b, c, *}$ \\ ${ }^{a}$ Division of Neural Dynamics, Department of System Neuroscience, National Institute for Physiological Sciences, National \\ Institutes of Natural Sciences, Okazaki 444-8585, Japan \\ ${ }^{b}$ Department of Physiological Sciences, School of Life Science, Graduate University for Advanced Studies (SOKENDAI), \\ Okazaki 444-8585, Japan \\ ${ }^{c}$ CBS-TOYOTA Collaboration Center, RIKEN Center for Brain Science, Wako, 351-0198, Japan
}

\begin{abstract}
Recent neuroscience studies have suggested that cognitive functions and learning capacity are reflected in the time-evolving dynamics of brain networks. However, an efficient method to detect changes in dynamical brain structures using neural data has yet to be established. To address this issue, we developed a new model-based approach to detect change points in dynamical network structures by combining the model-based network estimation with a phase-coupled oscillator model and sequential Bayesian inference. By giving the model parameter as the prior distribution, applying Bayesian inference allows the extent of temporal changes in dynamic brain networks to be quantified by comparing the prior distribution with posterior distribution using information theoretical criteria. For this, we used the Kullback-Leibler divergence as an index of such changes. To validate our method, we applied it to numerical data and electroencephalography data. As a result, we confirmed that the Kullback-Leibler divergence only increased when changes in dynamical network structures occurred. Our proposed method successfully estimated both directed network couplings and change points of dynamical structures in the numerical and electroencephalography data. These results suggest that our proposed method can reveal the neural basis of dynamic brain networks.
\end{abstract}

Keywords: Bayesian inference; change-point detection; Kullback-Leibler divergence; phase-coupled oscillator model; electroencephalography

\section{Introduction}

The ability to detect temporal changes of network dynamics from neural data has become of utmost importance for elucidating the role of neural dynamics in the brain. For instance, many neuroscience studies on the functional role of network dynamics in the brain have discussed the relevance of nonlinear coupled dynamical systems (Penny et al., 2009; Breakspear et al., 2010; Cabral et al., 2011; Deco et al., 2017b,a; Sase \& Kitajo, 2021). In addition, some recent experimental studies have raised the possibility that flexible changes in dynamic brain networks could be associated with cognitive functions and learning capacity (Bassett et al., 2011; Baum et al., 2017; Braun et al., 2015, 2016). However, an efficient method to detect changes in dynamical brain structures using neural data has yet to be established.

To detect temporal changes in brain networks in a data-driven manner, both temporal dependence in time-series data and structural relationships within the whole brain should be considered. In general, multivariate time-series data with non-stationary dynamics, such as neural data, not only contain information about structural relationships between dataset, but also about temporal dependence (i.e., the

${ }^{*}$ Division of Neural Dynamics, Department of System Neuroscience, National Institute for Physiological Sciences, National Institutes of Natural Sciences, Okazaki 444-8585, Japan

Email addresses: yokoyama@nips.ac.jp (Hiroshi Yokoyama), kkitajo@nips.ac.jp (Keiichi Kitajo) 
causal relationship from past to current states). Most previous studies have evaluated time-varying functional connectivity by applying the sliding window method (Hutchison et al., 2013; Damaraju et al., 2014). In this method, time series data are transformed into short epochs, each of which is analyzed separately and without explicitly dealing with the time-evolving dynamics. In most studies that have applied functional connectivity analysis, when evaluating the network structure within each time window, the coupling strength for each pair of brain areas is calculated based on the pairwise correlation between the time-series data. According to prior evidence from an economic study (Granger et al., 1974), correlation coefficients between two different time-series data with a high auto-correlation tend to be large, regardless of the existence of actual causality between the two time-series data. Moreover, since functional connectivity analysis considers the pairwise relationship of two different time series for each pair of brain regions, the resulting networks can easily lead to incorrect network couplings caused by the common drive from a third, confounding variable. Therefore, the conventional method of functional connectivity analysis cannot efficiently quantify the dynamical properties underlying temporal changes in neuronal activity. One possible approach to avoid the above-mentioned issues is to utilize a dynamical model-based network analysis. Namely, applying a coupled dynamical model for brain network analysis would allow the consideration of both network couplings and time-evolving dynamics reflected in neuronal time-series data. Given that neural data contain a strong temporal dependence in time-evolving dynamics, as well as network coupling, making the assumption that a dynamical system underlies neural data could be an essential way to address the aforementioned issues associated with functional connectivity analyses. As an additional advantage, this method would also reduce spurious network couplings, because a model-based analysis can differentiate between direct and indirect couplings.

However, when using a model-based approach, it is important to select a model that can estimate network couplings, because the model must be able to accurately explain the dynamical properties of the observed time-series data. To address the above issues, we developed a novel model-based method to detect temporal changes in dynamic brain networks that combines sequential Bayesian inference and dynamical model-based network analysis. Based on studies on nonlinear brain dynamics (Penny et al., 2009; Breakspear et al., 2010; Cabral et al., 2011; Deco et al., 2017b,a), we assumed that the time-evolving dynamics and the data structures of brain networks could be expressed as a phase-coupled oscillator model in this proposed method (Netoff et al., 2012; Penny et al., 2009; Ota \& Aoyagi, 2014; Suzuki et al., 2018; Onojima et al., 2018). Moreover, to capture the time-varying changes in network dynamics, our method recursively fitted the observed time series data to the phase-coupled oscillator model using sequential Bayesian inference (Sarris, 1973; Bishop, 2007). The advantage of using sequential Bayesian inference for a model fitting is that, by giving the model parameter as the prior distribution, the extent of temporal changes in the brain network can be quantified based on the comparison between prior and posterior distributions using information theoretical criteria. Considering this, we predicted that temporal changes in dynamical structures of the brain network would be reflected in the change in model parameters. Therefore, to define an index for the change in dynamical structures of functional brain networks, the difference between prior and posterior distributions of model parameters was measured using the Kullback-Leibler (KL) divergence.

To validate our proposed method, two examinations were performed. First, we applied our method to a time series of synthetic data with known dynamical changes of network coupling, which were generated by a weakly phase-coupled oscillator model. Doing so allowed us to confirm whether the change points detected by our proposed method were consistent with the exact timing of network coupling changes in the synthetic data. Next, we applied our method to an open dataset of real electroencephalography (EEG) signals measured in healthy human participants who were passively listening to binaural auditory stimuli of 40-Hz amplitude-modulated (AM) white noise (McFadden et al., 2014). In this way, we assessed whether our proposed method could detect the timing of changes in phase-coupled EEG networks induced by the periodic auditory stimuli. 


\section{Materials and methods}

In this section, we will first provide the theoretical background for the phase-coupled oscillator model and the details of our proposed method. We will then explain the procedures for model validation using synthetic data from numerical models and EEG data.

\subsection{Dynamical systems theory of the phase oscillator model}

In our proposed method, to estimate the dynamical structure in the brain networks, we applied a data-driven modeling method that uses phase-response curves (Kuramoto, 1984; Ko \& Ermentrout, 2009; Netoff et al., 2012; Stankovski et al., 2017; Pietras \& Daffertshofer, 2019). From the point of view of dynamical systems, the time series $\boldsymbol{X} \in \mathbb{R}^{\mathrm{N}}$, which is followed by a periodic function $F$, can be described as follows:

$$
\frac{d \boldsymbol{X}}{d t}=F(\boldsymbol{X})
$$

Here, we consider two coupled oscillators, $\boldsymbol{X}=\left\{x_{i}, x_{j}\right\}$, driven by an interaction between oscillators. In this case, the dynamics can be described as follows:

$$
\frac{d x_{i}}{d t}=F_{i}\left(x_{i}\right)+g_{i j}\left(x_{j}, x_{i}\right),
$$

where $F_{i}\left(x_{i}\right)$ and $g_{i j}\left(x_{j}, x_{i}\right)$ indicate the dynamical system function of the $i$-th oscillator and the interaction between $i$ and $j$-th oscillators, respectively.

In the polar coordinates, if the system exhibits limit cycles with a stable point, Eq. (2) can be theoretically described by a phase $\phi$ as a simple dynamical system with one degree of freedom (Kuramoto, 1984; Pietras \& Daffertshofer, 2019; Onojima et al., 2018; Suzuki et al., 2018; Ota \& Aoyagi, 2014) (See the text in Appendix A for more details).

$$
\frac{d \phi_{i}}{d t}=\omega+\Gamma_{i j}\left(\phi_{j}-\phi_{i}\right)
$$

where $\omega$ and $\Gamma_{i j}\left(\phi_{j}-\phi_{i}\right)$ indicate the natural frequency and phase interaction function from $j$-th to $i$-th oscillator. Given the above equations, the function $\Gamma_{i j}\left(\phi_{j}-\phi_{i}\right)$ describes the phase changes in the cycle period of coupled oscillators in terms of the network interactions for each oscillator. Thus, by estimating the function $\Gamma_{i j}\left(\phi_{j}-\phi_{i}\right)$ from empirically measured data, we can characterize the interaction between oscillators. Hereafter, we call this function $\Gamma_{i j}\left(\phi_{j}-\phi_{i}\right)$ as the "phase interaction function" (Penny et al., 2009; Ota \& Aoyagi, 2014; Suzuki et al., 2018; Onojima et al., 2018).

\subsection{Phase oscillator model approximated with the Fourier series}

Considering the phase oscillator model theory described in section 2.1, we applied the following equation to describe the dynamical network model in the brain:

$$
\frac{d \phi_{i}(t)}{d t}=\omega_{i}(t)+\Gamma_{i j}\left(\phi_{j}(t)-\phi_{i}(t)\right)+\eta_{i},
$$

where $\phi_{i}(t), \omega_{i}(t)$, and $\eta_{i}(t)$ indicate the phase, the natural frequency, and the observation noise of the $i$-th oscillator, respectively. In addition, some computational and methodological neuroscience studies (Penny et al., 2009; Netoff et al., 2012; Ota \& Aoyagi, 2014; Suzuki et al., 2018; Onojima et al., 2018) have proposed the methods to estimate the function $\Gamma_{i j}\left(\phi_{j}-\phi_{i}\right)$ of neural data. In these studies, the function $\Gamma_{i j}\left(\phi_{j}-\phi_{i}\right)$ is approximated as the $P$-th Fourier series (Penny et al., 2009; Netoff et al., 2012; Ota \& Aoyagi, 2014; Suzuki et al., 2018; Onojima et al., 2018), because the function $\Gamma_{i j}\left(\phi_{j}-\phi_{i}\right)$ is a $2-\pi$ periodic function. Therefore, we also applied the $P$-th Fourier series to estimate the function $\Gamma_{i j}\left(\phi_{j}-\phi_{i}\right)$, and its parameters in the Fourier series were estimated by fitting the observed data to Eq. (5) using Bayesian inference. In our study, Eq. (4) was approximated as follows: 


$$
\begin{aligned}
& \frac{d \phi_{i}(t)}{d t}=\hat{\omega}_{i}(t)+\sum_{p=1}^{P} \sum_{j \neq i}^{N}\left\{a_{i j}^{(p)}(t) \cos \left[p\left(\phi_{j}(t)-\phi_{i}(t)\right)\right]\right. \\
& \left.\quad+b_{i j}^{(p)}(t) \sin \left[p\left(\phi_{j}(t)-\phi_{i}(t)\right)\right]\right\}+\eta_{i}(t), \\
& \text { where } \quad \hat{\omega}_{i}(t)=\omega_{i}(t)+\sum_{j \neq i}^{N} a_{i j}^{(0)}(t) .
\end{aligned}
$$

Note that $a_{i j}^{(p)}$ and $b_{i j}^{(p)}$ indicate the coefficients of the $p$-th Fourier series corresponding to the coupling strength between the $i$ and $j$-th oscillators. Moreover, $\hat{\omega}_{i}(t)$ is the neutral frequency of this model. In our proposed method, the coupling strength $\kappa_{i j}$ for all $i, j$ is defined as follows:

$$
\kappa_{i j}(t)=\sqrt{\sum_{p=1}^{P}\left\{a_{i j}^{(p)}(t)\right\}^{2}+\left\{b_{i j}^{(p)}(t)\right\}^{2}} .
$$

\subsection{Estimation of the model parameters and change point scores}

We will next explain how to predict the model parameters shown in Eq. (5) and the main idea behind change point detection in observed data. To evaluate the temporal changes in the networks, we extended the parameter estimation algorithm in Eq. (5) using Bayesian inference (Bishop, 2007; Sarris, 1973). In this way, the parameters of Eq. (5) could be directly estimated from the observed data in the same way as for sequential Bayesian regression. To apply Bayesian inference, Eq. (5) was rewritten in its matrix form, as shown in Eq. (7) .

$$
\mathbf{\Phi}^{\prime}=F(\boldsymbol{\Phi}, \boldsymbol{K})+\eta=\boldsymbol{f} \cdot \boldsymbol{K}+\eta
$$

where,

$$
\begin{gathered}
\boldsymbol{\Phi}^{\prime}=\left[\frac{\phi_{1}(t+d t)-\phi_{1}(t)}{d t}, \ldots, \frac{\phi_{N}(t+d t)-\phi_{N}(t)}{d t}\right], \\
\boldsymbol{f}=\left[\begin{array}{cccc}
f_{1}(\boldsymbol{\Phi}, t) & O & \ldots & O \\
O & \boldsymbol{f}_{2}(\Phi, t) & \ldots & O \\
\vdots & \vdots & \ddots & \vdots \\
O & O & \ldots & \boldsymbol{f}_{N}(\Phi, t)
\end{array}\right] \\
\boldsymbol{f}_{i}(\boldsymbol{\Phi}, t)=\left\{\begin{array}{c}
\cos \left[\phi_{1}(t)-\phi_{i}(t)\right], \ldots, \cos \left[\phi_{N}(t)-\phi_{i}(t)\right], \\
\sin \left[\phi_{1}(t)-\phi_{i}(t)\right], \ldots, \sin \left[\phi_{N}(t)-\phi_{i}(t)\right], \ldots \ldots, \\
\cos \left[P\left(\phi_{1}(t)-\phi_{i}(t)\right)\right], \ldots, \cos \left[P\left(\phi_{N}(t)-\phi_{i}(t)\right],\right. \\
\sin \left[P\left(\phi_{1}(t)-\phi_{i}(t)\right)\right], \ldots, \sin \left[P\left(\phi_{N}(t)-\phi_{i}(t)\right], 1\right\} \\
\boldsymbol{K}=\left[\boldsymbol{K}_{1}(t), \boldsymbol{K}_{2}(t), \ldots, \boldsymbol{K}_{N}(t)\right]^{T}, \\
\boldsymbol{K}_{i}(t)=\left\{a_{i 1}^{(1)}(t), \ldots, a_{i N}^{(1)}(t), b_{i 1}^{(1)}(t), \ldots, b_{i N}^{(1)}(t), \ldots \ldots,\right. \\
\left.a_{i 1}^{(P)}(t), \ldots, a_{i N}^{(P)}(t), b_{i 1}^{(P)}(t), \ldots, b_{i N}^{(P)}(t), \hat{\omega}_{i}(t)\right\} .
\end{array}\right.
\end{gathered}
$$

Note that $F(\boldsymbol{\Phi}, \boldsymbol{K})$ is the function of phase-coupled network dynamics characterized by phase vector $\phi$ and the parameter vector $\boldsymbol{K}$. In our model, $F(\boldsymbol{\Phi}, \boldsymbol{K})$ and $\boldsymbol{K}$ indicate the Fourier series and its coefficient, respectively. Moreover, $\boldsymbol{\Phi}^{\prime}$ indicates a time derivative of the phase with a smaller time step $d t$. In the 
following simulations (see section 2.4 for more details), we set the time step as $d t=0.01$. Moreover, for the real measured data, the time step $d t$ corresponds to the sampling interval of the data.

Given the prior distribution of the model parameters, the parameter $\boldsymbol{K}$ in Eq. (7) can be calculated using the Bayesian rule (Bishop, 2007; Sarris, 1973), as follows:

$$
\begin{aligned}
& \boldsymbol{\Phi}^{\prime}=\boldsymbol{f} \cdot \boldsymbol{K}+\eta, \quad \eta \sim \mathcal{N}\left(0, \beta^{-1} I\right), \\
& \boldsymbol{\Phi}^{\prime} \sim p\left(\boldsymbol{\Phi}^{\prime}=\frac{\boldsymbol{\Phi}(t+d t)-\boldsymbol{\Phi}(t)}{d t} \mid \boldsymbol{f}, \boldsymbol{K}\right), \\
& p\left(\boldsymbol{K} \mid \boldsymbol{f}, \boldsymbol{\Phi}^{\prime}\right) \propto p\left(\boldsymbol{\Phi}^{\prime}=\frac{\boldsymbol{\Phi}(t+d t)-\boldsymbol{\Phi}(t)}{d t} \mid \boldsymbol{f}, \boldsymbol{K}\right) p(\boldsymbol{K}) .
\end{aligned}
$$

Here, in our proposed method, the prior distribution $p(\boldsymbol{K})$ is assumed to have a multivariate normal distribution $\mathcal{N}\left(\boldsymbol{\mu}_{\boldsymbol{K}}, \boldsymbol{\Sigma}_{\boldsymbol{K}}\right)$, where $\boldsymbol{\mu}_{\boldsymbol{K}}$ and $\boldsymbol{\Sigma}_{\boldsymbol{K}}$ indicate the mean and covariance of the distribution, respectively. The parameter $\beta$ is a precision factor of the observation noise, and this parameter is an arbitrary constant term. In this study, this parameter was fixed as $\beta^{-1}=0.0001$ for all analyses.

The update rule of the prior distribution is derived as follows (Sarris, 1973):

$$
\begin{aligned}
& \overline{\boldsymbol{\Sigma}}_{\boldsymbol{K}}=\boldsymbol{\Sigma}_{\boldsymbol{K}}-\boldsymbol{\Sigma}_{\boldsymbol{K}} \boldsymbol{f}^{T}\left(\beta^{-1} I+\boldsymbol{f} \boldsymbol{\Sigma}_{\boldsymbol{K}} \boldsymbol{f}^{T}\right)^{-1} \boldsymbol{f} \boldsymbol{\Sigma}_{\boldsymbol{K}} . \\
& \overline{\boldsymbol{\mu}}_{\boldsymbol{K}}=\boldsymbol{\mu}_{\boldsymbol{K}}+\boldsymbol{\Sigma}_{\boldsymbol{K}} \boldsymbol{f}^{T}\left(\beta^{-1} I+\boldsymbol{f} \boldsymbol{\Sigma}_{\boldsymbol{K}} \boldsymbol{f}^{T}\right)^{-1}\left(\boldsymbol{\phi}^{\prime}-\boldsymbol{f} \boldsymbol{\mu}_{\boldsymbol{K}}\right) .
\end{aligned}
$$

where $\overline{\boldsymbol{\mu}}_{\boldsymbol{K}}$ and $\overline{\boldsymbol{\Sigma}}_{\boldsymbol{K}}$ indicate the updated mean and covariance, respectively. After the update of the prior distribution, these parameters are set as $\boldsymbol{\mu}_{K}=\overline{\boldsymbol{\mu}}_{K}$ and $\boldsymbol{\Sigma}_{\boldsymbol{K}}=\overline{\boldsymbol{\Sigma}}_{\boldsymbol{K}}$ for the next updating process. equation:

Moreover, the posterior predictive distribution of the model can be inferred using the following

$$
\overline{\boldsymbol{\Phi}}^{\prime} \sim \mathcal{N}\left(\boldsymbol{f} \boldsymbol{\mu}_{\boldsymbol{K}}, \quad \beta^{-1} I+\boldsymbol{f} \boldsymbol{\Sigma}_{\boldsymbol{K}} \boldsymbol{f}^{T}\right),
$$

where $\overline{\boldsymbol{\Phi}}^{\prime}$ indicates the predicted phase velocity using the inferred model parameters. Based on the above procedure, the temporal changes in networks were estimated based on sequential Bayesian inference.

As a side note, by introducing the matrix $\boldsymbol{G}=\boldsymbol{\Sigma}_{\boldsymbol{K}} \boldsymbol{f}^{T}\left(\beta^{-1} I+\boldsymbol{f} \boldsymbol{\Sigma}_{\boldsymbol{K}} \boldsymbol{f}^{T}\right)^{-1}=\boldsymbol{\Sigma}_{\boldsymbol{K}} \boldsymbol{f}^{T} \boldsymbol{S}^{-1}$ and the covariance $\boldsymbol{S}=\beta^{-1} I+\boldsymbol{f} \boldsymbol{\Sigma}_{\boldsymbol{K}} \boldsymbol{f}^{T}$, Eq. (9) can be rewritten as follows:

$$
\begin{aligned}
& \bar{\Sigma}_{K}=\Sigma_{K}-G S G^{T}, \\
& \bar{\mu}_{K}=\mu_{K}+G\left(\Phi^{\prime}-f \mu_{K}\right) .
\end{aligned}
$$

This matrix $\boldsymbol{G}$ is called the Kalman gain. Therefore, the sequential form of Bayesian regression with the multivariate normal prior is a special case of the Kalman filter (Sarris, 1973).

The advantage of applying Bayesian inference to estimate the function $\Gamma_{i j}\left(\phi_{j}-\phi_{i}\right)$ of dynamical networks is that the current state of the network dynamics and its dynamical structure can be expressed using a probability distribution. This means that the extent of change in the network structures can be quantified based on the comparison between prior and posterior distributions of model parameters. From the viewpoint of change-point detection based on probability statistics, if the past and current state of variable $x$ can explain the probability density $p(x)$ and $q(x)$, the changes in variable $x$ are typically measured as the gap between $p(x)$ and $q(x)$ using the log-likelihood ratio (Basseville et al., 1993). Moreover, the expectation under the probability $p(x)$ of the $\log$-likelihood ratio between $p(x)$ and $q(x)$ can be described as the KL divergence (Basseville et al., 1993), as follows:

$$
E_{p(x)}\left\{\log \frac{p(x)}{q(x)}\right\}=\sum p(x) \log \frac{p(x)}{q(x)}=D_{K L}(p(x) \| q(x)) .
$$


Note that $E_{p(x)}\{\cdot\}$ indicates the expectation operator under the probability $p(x) . D_{K L}(p(x) \| q(x))$ indicates the KL divergence from $q(x)$ to $p(x)$. Based on these statistical backgrounds, we measured the extent of change in the network structures using the KL divergence between the prior and posterior probability density of the model parameters. Therefore, our proposed method used the KL divergence between the prior and posterior distribution as an index of the change in dynamical structures, as shown in Eq. (13), and the value of this KL divergence is defined as the change point score (CPS) as follows:

$$
\mathrm{CPS}=D_{K L}\left(\mathcal{N}\left(\overline{\boldsymbol{\mu}}_{\boldsymbol{K}}, \overline{\boldsymbol{\Sigma}}_{\boldsymbol{K}}\right) \| \mathcal{N}\left(\boldsymbol{\mu}_{\boldsymbol{K}}, \boldsymbol{\Sigma}_{\boldsymbol{K}}\right)\right) .
$$

where $\boldsymbol{\mu}_{K}$ and $\overline{\boldsymbol{\mu}}_{K}$ indicate the mean of the parameter $K$ for the prior and posterior distribution, respectively. Also, $\boldsymbol{\Sigma}_{\boldsymbol{K}}$ and $\overline{\boldsymbol{\Sigma}}_{\boldsymbol{K}}$ indicate the covariance of the parameter $K$ for the prior and posterior distribution, respectively. Using the above process, we can detect change points in network dynamics while sequentially estimating dynamical structures in the brain using the KL divergence value. Fig. 1 shows an overview of the change point detection in our proposed method.

$$
\begin{aligned}
& \text { Phase velocity } \\
& \text { Network dynamics : } \\
& \Phi^{\prime}(t)=\frac{F(\Phi(t), K(t))+\eta(t)}{\mathrm{L} \text { coupled phase oscillator model }}
\end{aligned}
$$

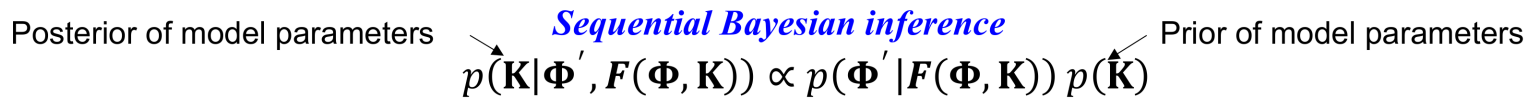

Change-point detection

$$
\left.\begin{array}{r}
p(\mathbf{K})=\mathcal{N}\left(\boldsymbol{\mu}_{\mathrm{K}}, \mathbf{\Sigma}_{\mathrm{K}}\right) \\
p\left(\mathbf{K} \mid \Phi^{\prime}, \mathbf{f}(\boldsymbol{\Phi}, \mathbf{K})\right)=\mathcal{N}\left(\overline{\boldsymbol{\mu}}_{\mathrm{K}}, \overline{\boldsymbol{\Sigma}}_{\mathrm{K}}\right)
\end{array}\right\} \text { Compare between } \mathcal{N}\left(\boldsymbol{\mu}_{\mathrm{K}}, \boldsymbol{\Sigma}_{\mathrm{K}}\right) \text { and } \mathcal{N}\left(\overline{\boldsymbol{\mu}}_{\mathrm{K}}, \overline{\boldsymbol{\Sigma}}_{\mathrm{K}}\right)
$$

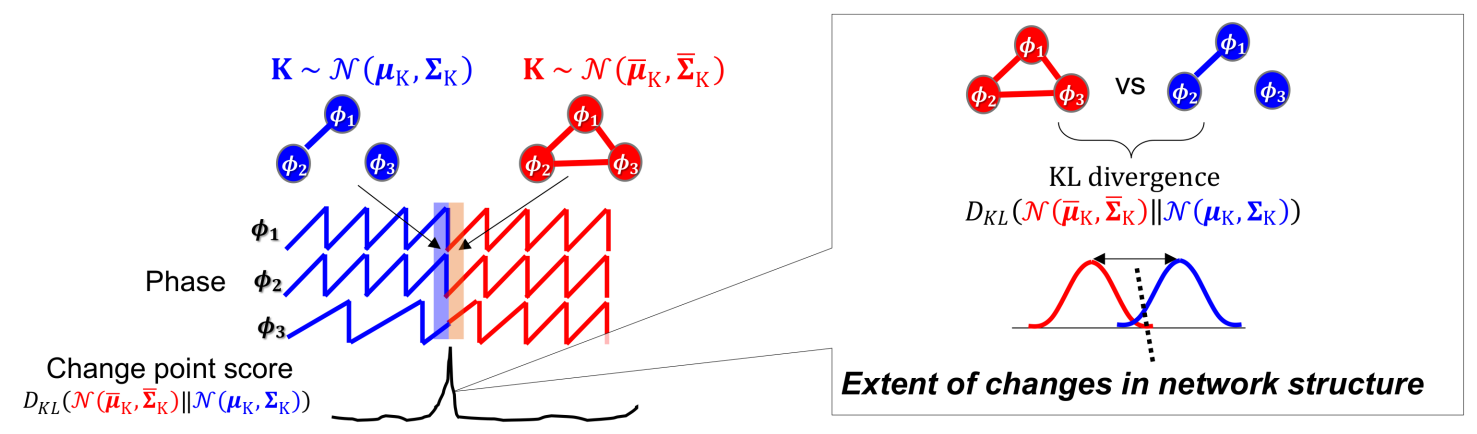

Assumption: "Changes in the network structure" $=$ "Changes in the prior distribution"

Figure 1: Overview of our proposed method for network change point detection. In our proposed method, the time series data are fitted with a phase-coupled oscillator model using Bayesian inference. We here assume that network changes are represented as the changes to the model parameters regarding coupling strength between phase oscillators. By giving the prior distribution of the model parameters, the structural changes in networks can be quantified by comparing prior and updated posterior distributions. Therefore, the changes in dynamical structures of brain networks are quantified using the Kullback-Leibler divergence between prior and posterior distributions. 
In summary, our proposed method consists of the following seven steps. A schematic view of the following steps is shown in Fig. 2.

1. Give the initial value of $\boldsymbol{\mu}_{\boldsymbol{K}}$ and $\boldsymbol{\Sigma}_{\boldsymbol{K}}$ for the prior distribution of the model parameter

2. Get new single samples for each phase $\boldsymbol{\Phi}(t)$, and calculate new variable $\boldsymbol{f}$ and $\boldsymbol{\Phi}^{\prime}(t)$

3. Update predictive distribution of the model (see Prediction step in Fig. 2)

4. Update the prior's parameters (see Update step in Fig. 2)

5. Calculate the KL divergence between prior and posterior model parameters (i.e., evaluate the change point score)

6. Substitute parameters as $\boldsymbol{\mu}_{\boldsymbol{K}}=\overline{\boldsymbol{\mu}}_{\boldsymbol{K}}$ and $\boldsymbol{\Sigma}_{\boldsymbol{K}}=\overline{\boldsymbol{\Sigma}}_{\boldsymbol{K}}$ for the next Prediction step

7. Repeat steps 2 to 6

According to the above-mentioned description, the model parameter updating process is conducted on a sample-by-sample basis, and the metric of change point score using the KL divergence is also calculated after updating the prior of the model parameters sample-by-sample.

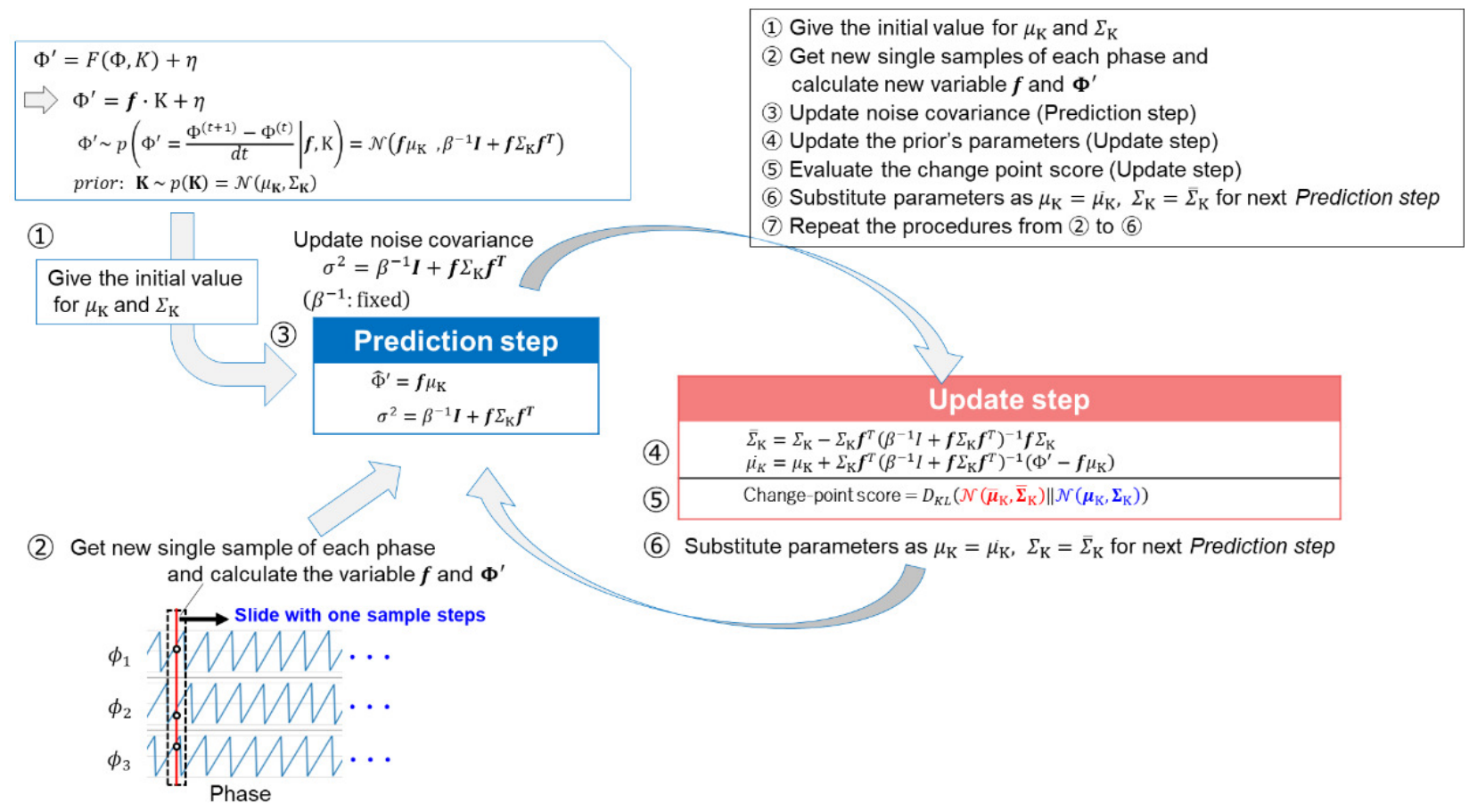

Figure 2: Schematic view of the sequential Bayesian inference algorithm. First, after giving the initial values of $\mu_{\mathrm{K}}$ and $\Sigma_{\mathrm{K}}$ for the prior distribution, the predictive posterior distribution of the model is updated with the sequentially sampled data $\boldsymbol{f}$ and $\boldsymbol{\Phi}^{\prime}$ ("Prediction step"), and the noise covariance $\sigma^{2}$ is updated. Next, the prior distribution of the model parameters is updated ("Update step"). After this, the extent of change in dynamical structures of the network is assessed using the Kullback-Leibler divergence (i.e., evaluation of the change point score). The updated parameters of the prior distribution are set as $\mu_{\mathrm{K}}=\bar{\mu}_{\mathrm{K}}$ and $\Sigma_{\mathrm{K}}=\bar{\Sigma}_{\mathrm{K}}$ for the next "Prediction step". According to the above-mentioned description, the model parameter updating process is conducted on a sample-by-sample basis, and the metric of change point score using the KL divergence is also calculated after updating the prior of the model parameters sample-by-sample. 


\subsection{Validation of the proposed method}

\subsubsection{Generation of synthetic data}

In all simulations, which are described in the section 2.4.2 to 2.4.4, we used the stochastic phase-coupled oscillator model approximated with a first-order Fourier series to generate the synthetic data. The used model is defined as follows:

$$
\begin{aligned}
& \frac{d \phi_{i}(t)}{d t}=\hat{\omega}_{i}(t)+\sum_{j \neq i}^{N}\left\{a_{i j}(t) \cos \left(\phi_{j}(t)-\phi_{i}(t)\right)+b_{i j}(t) \sin \left(\phi_{j}(t)-\phi_{i}(t)\right)\right\}+p_{i} d W_{i}, \\
& \text { where } \hat{\omega}_{i}(t)=\omega_{i}(t)+\sum_{j \neq i}^{N} a_{i j}^{(0)}(t), \\
& \qquad d W_{i} \sim \sqrt{d t} \mathcal{N}(0,1),
\end{aligned}
$$

where $p_{i}$ and $d W_{i}$ indicate the noise scaling factor and white noise factor, which followed a normal distribution $\sqrt{d t} \mathcal{N}(0,1)$. The synthetic data were generated using the numerical integration of Eq. (14) using the Euler-Maruyama method. The integration scheme of the Euler-Maruyama method in Eq. (14) is repetitively conducted using the following equation:

$$
\phi_{i}(t+d t)=\phi_{i}(t)+\left(\hat{\omega}_{i}(t)+\sum_{j \neq i}^{N}\left\{a_{i j}(t) \cos \left(\phi_{j}(t)-\phi_{i}(t)\right)+b_{i j}(t) \sin \left(\phi_{j}(t)-\phi_{i}(t)\right)\right\}\right) d t+p_{i} \sqrt{d t} \mathcal{N}(0,1),
$$

where $d t$ indicates the time step. As mentioned above, in the simulations, the time step is set as $d t=0.01$. Other parameter, such as Fourier coefficient $a_{i j}, b_{i j}$, natural frequency $\hat{\omega}_{i}$, and noise scaling factor $p_{i}$, were set as the different values depending on the settings for each simulation (see the section 2.4.2 to 2.4.4 for more details) .

\subsubsection{Numerical simulation $A$}

To validate our proposed method, we first applied it to synthetic time-series data generated by N-coupled oscillators with fixed network couplings. The purpose of this simulation was to clarify the number of practical oscillators and the number of learning iterations of the model required to accurately estimate the network couplings when using our proposed method.

In this simulation, we set four conditions of $N_{\text {osci }}=[3,10,15,20,30]$, and generated 4,000 samples of synthetic data for each condition of $N_{o s c i}$ using Eq. (15). The parameter settings of Fourier coefficients $a_{i j}$ and $b_{i j}$ for each condition were selected, as shown in Fig. 3. Moreover, the natural frequency $\hat{\omega}_{i}=2 \pi f_{i}$ of the $i$-th oscillator is set as a fixed value so that $f_{i}$ follows a normal distribution $\mathcal{N}(4.0,0.5)$. The noise scaling factor $p_{i}$ for the integration scheme in Eq. (15) was set as $p_{i}=0.001$.

After generating the synthetic data using Eq. (15) with the above parameter settings, our Bayesian inference-based method was applied to the time series of synthetic phase data. The precision parameter $\beta$ in Eqs. (9) and (10) was fixed at $\beta^{-1}=0.0001$ for this simulation. For each iteration of the model updating steps, we evaluated the error of the structure of network couplings with the mean absolute error (MAE) to evaluate the estimation accuracy, as follows:

$$
M A E=\frac{1}{\# \text { pairs of oscillators }} \sum_{i, j}\left|K_{i j}-\hat{K}_{i j}(t)\right|
$$

where $K_{i j}$ indicates the exact coupling strength value between the pair of oscillators $i, j$ in the synthetic data, and $\hat{K}_{i j}(t)$ indicates the estimated value of coupling strength between the $i$-th and $j$-th oscillators in time $t$. The value of $\hat{K}_{i j}(t)$ in time $t$ were calculated using estimated Fourier coefficients $\hat{a}_{i j}(t)$ and $\hat{b}_{i j}(t)$ predicted by sequential Bayesian inference, as shown in Fig. 2. 
To calculate the confidence intervals of estimation error of the coupling strength $\hat{K}_{i j}(t)$, the surrogate method was applied. Specifically, to generate the null distribution of the randomized MAE, we first calculated surrogate $\hat{K}_{i j}(t)$, in which the the temporal order of the estimated $\hat{K}_{i j}(t)$ is shuffled 1,000 times, and the MAE between the true network coupling strength $K_{i j}$ and the shuffled $\hat{K}_{i j}(t)$ was calculated. Then, the resulting values of these surrogate MAEs were used to compose the null distribution of MAEs, and to assess the $95 \%$ confidence intervals of the estimation error.

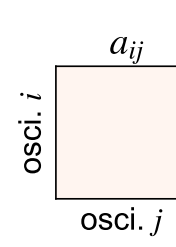

$$
N_{\text {osci }}=3
$$
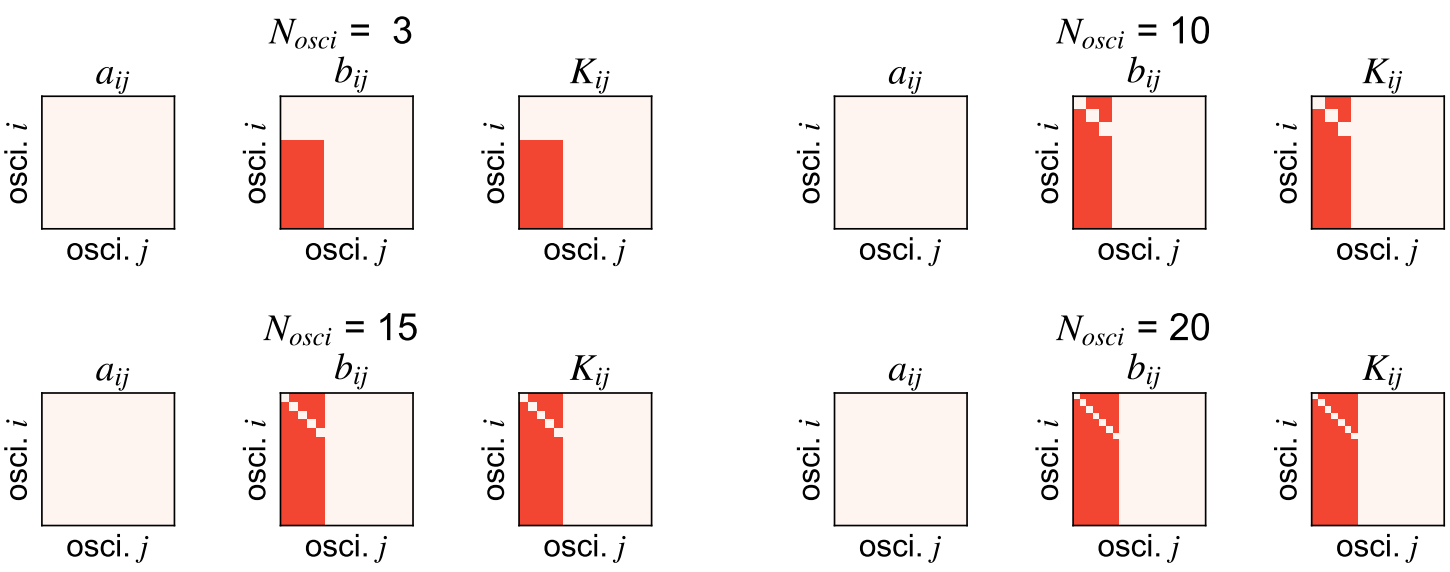

$$
N_{\text {osci }}=15
$$
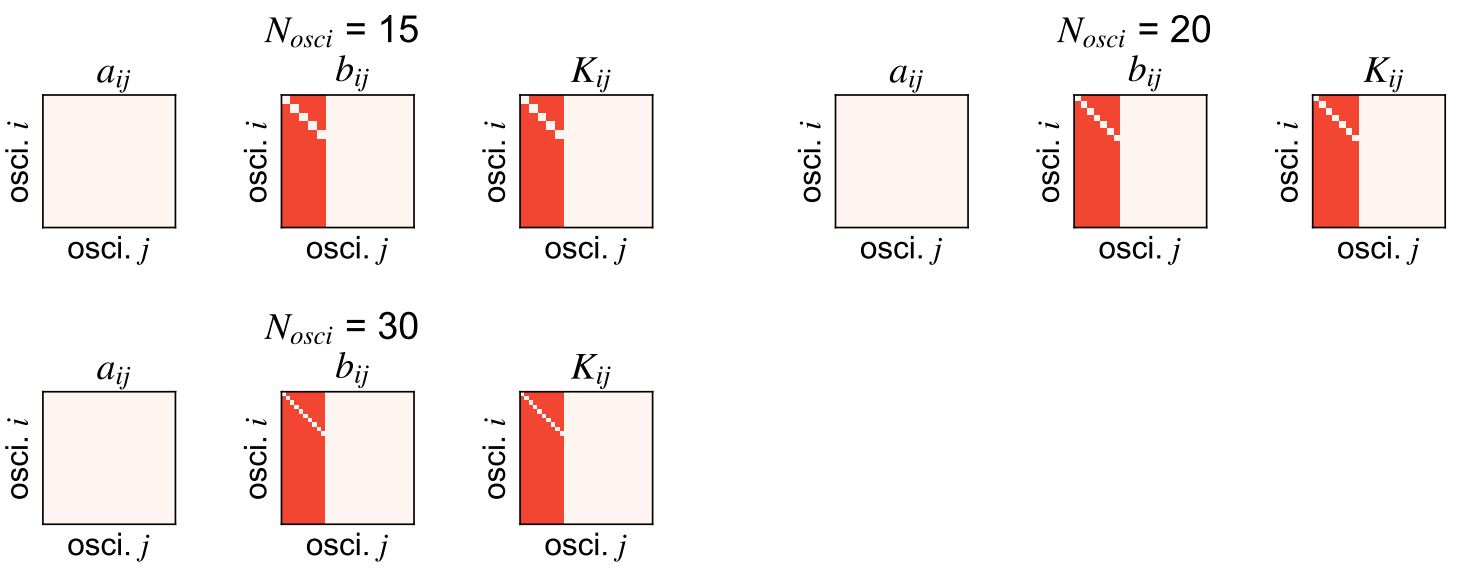

$$
-0.4
$$

$-0.2$

\section{0}

0.2

0.4

Figure 3: Parameter settings in numerical simulation A. This simulation set five different conditions for the number of oscillators $\left(N_{\text {osci }}=3,10,15,20\right.$, and 30). For each condition, the time series of synthetic data in this simulation were generated by a first-order Fourier series-approximated phase-coupled oscillator model with the parameter settings shown in this figure. The Fourier coefficients $a_{i j}, b_{i j}$ and coupling strength $K_{i j}$ were selected such that one-third of oscillator pairs interacted. 


\subsubsection{Numerical simulation $B$}

The first numerical simulation revealed that the practical number of oscillators to which our proposed method can be applied is less than 10 (see the "Results" section for details). Considering this, we conducted a numerical simulation using synthetic data generated by an N-coupled oscillator model (N: less than 10) to clarify whether our method can sequentially detect changes in the network couplings.

First, we applied our method to 3,000 samples of synthetic time series data of three coupled oscillators generated by Eq. (15) with a first-order Fourier series. These synthetic time series consisted of three segments separated by two events. In the first event, the Fourier coefficients $a_{i j}$ and $b_{i j}$ changed, which indicated the occurrence of changes in the dynamical structure of networks in the boundary between segments 1 and 2 (hereafter, this event is called "structural change"). In the second event, the Fourier coefficients $a_{i j}$ and $b_{i j}$ did not change between segments 2 and 3 ; however, the observation noise, which does not directly contribute to temporal dynamics, drastically changed (hereafter, this event is called "noise-level change"). The exact values of the parameters $a_{i j}$ and $b_{i j}$ were set as shown in Fig. 4A. The natural frequency $\hat{\omega}_{i}=2 \pi f_{i}$ of oscillator $i$ was set at a fixed value so that $f_{i}$ followed a normal distribution $\mathcal{N}(4.0,0.5)$. Moreover, the noise-scaling factor $p_{i}$ for each $i$-th oscillator in Eq. (15) was set as follow:

$$
p_{i}= \begin{cases}0.001 & :(\text { segment } 1, \text { segment } 2) \\ 0.01 & :(\text { segment } 3) .\end{cases}
$$

After generating the synthetic data using Eq. (15) with the above parameter settings, our method was applied to these synthetic data as follows:

- The time series of synthetic data was fit to the first-order Fourier series using Bayesian inference (Fig. 2)

- The KL divergence between prior and posterior model parameters was evaluated (i.e., we calculated the change point score; Figs. 1 and 2)

- The time-sample with a greater change point score relative to the threshold was selected as the "change point"

Note that the threshold for the change point score was set as the mean $(\mathrm{KL})+3 \mathrm{SD}(\mathrm{KL})$; these terms refer to the mean KL divergence and 3 standard deviations of the KL divergence of the change point score across samples, respectively. The precision parameter $\beta$ in Eqs. (9) and (10) was fixed at $\beta^{-1}=0.0001$ for this simulation. 
A
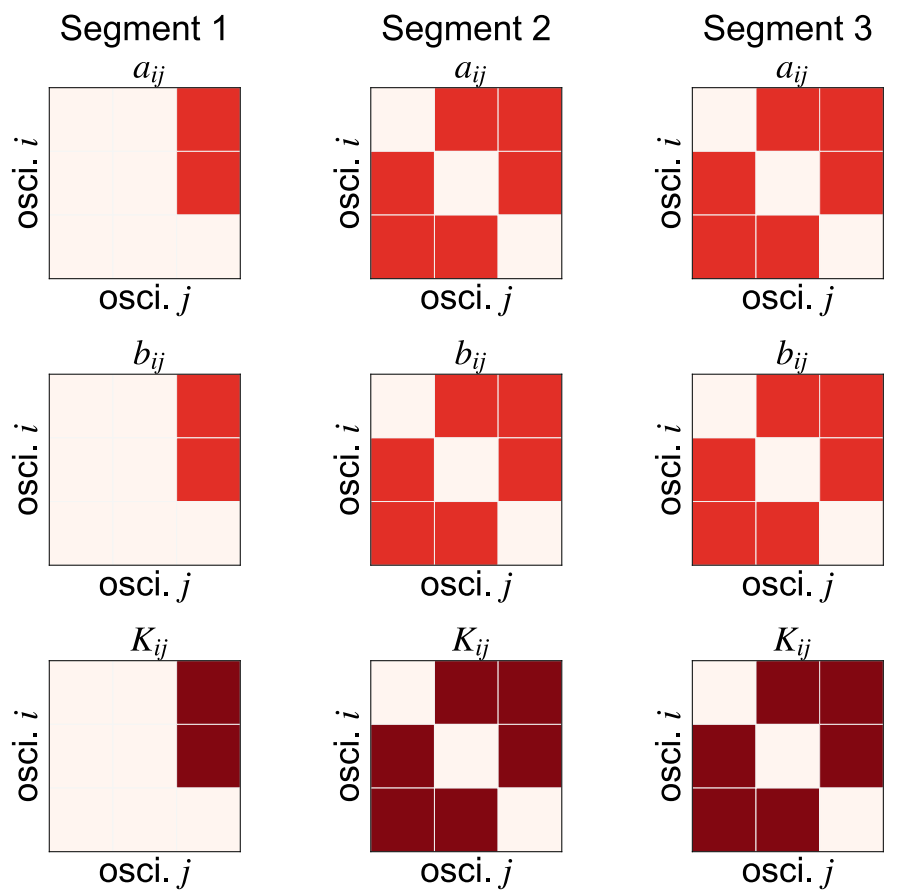

$K_{i j}$

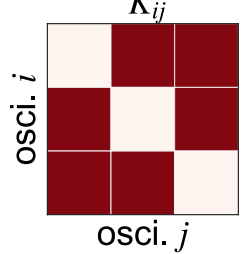

Segment 3

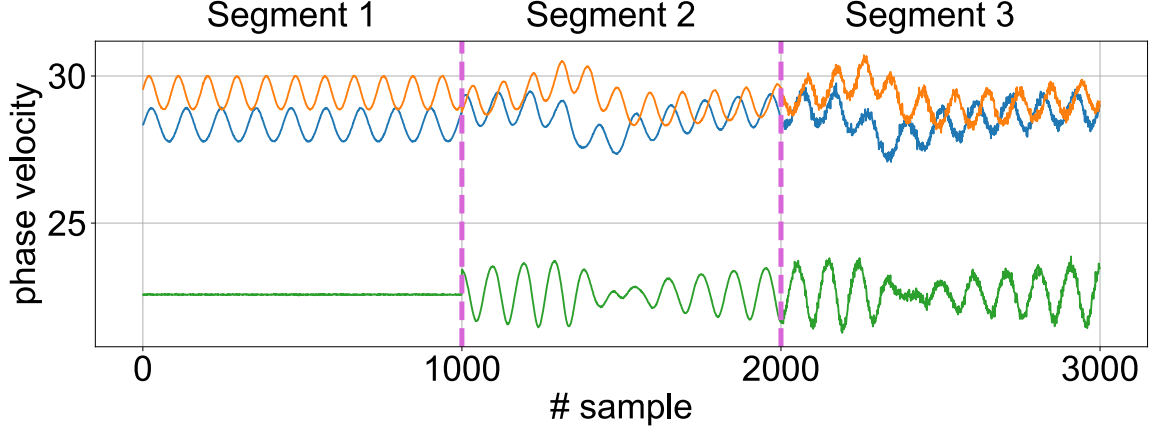

oscillator 1 oscillator 2 oscillator 3

Figure 4: Parameter settings in numerical simulation B. (A) True parameter settings in each segment. The time series of synthetic data was generated using the function $\Gamma_{i j}\left(\phi_{j}-\phi_{i}\right)$ approximated by the first-order Fourier series using these parameter settings in each segment. (B) This panel shows the temporal behavior of the synthetic phase-oscillator model with the above-mentioned parameter settings. The figure shows synthetic data of three oscillators generated by Eqs. (14) and (15) with the first-order Fourier series. These synthetic time series consisted of three segments separated by two events (see the text for the details). The pink dashed lines mark the first and second events. 
Next, to compare our method with the conventional method, the network changes in synthetic data were also evaluated using the Leading Eigenvector Dynamics Analysis (LEiDA) algorithm, which was proposed by Cabral et al. (2017). The LEiDA algorithm is configured using the following three steps. In the first step, to evaluate temporal changes in phase coherence for each pair of oscillators, the following value, called dynamic functional connectivity $(\mathrm{dFC})$, is evaluated for each time point as follows:

$$
d F C_{i j}(t)=\cos \left(\phi_{i}(t)-\phi_{j}(t)\right)
$$

where $i, j$ indicates the indices of the oscillators, and $\phi_{i}(t)$ represents the phase of the $i$-th oscillator at time point $t$. Using this equation, we obtained the $\mathrm{dFC}$, which is the three-dimensional tensor with the size of $N_{o s c i} \times N_{o s c i} \times N_{t}$. Note that $N_{o s c i}$ and $N_{t}$ indicate the number of oscillators and the number of time samples, respectively. In the second step, to reduce the dimensionality of such a dFC matrix, the eigenvalue decomposition was applied to the $\mathrm{dFC}(\mathrm{t})$ with a size of $N_{\text {osci }} \times N_{\text {osci }}$ for each time point $t$, and the first eigenvector $V_{1}(t)$, corresponding to the eigenvalue with the largest magnitude, was selected for each time point. We considered the vector $V_{1}(t)$ with a size of $N_{o s c i} \times 1$ to reflect the dominant pattern of the functional connectivity for each time point. Therefore, the $\mathrm{dFC}$ matrix at time point $t$ can be reconstructed using the inner product of this eigenvector for each time point, such as $V_{1}(t) V_{1}^{T}(t)$. The resulting eigenvectors $V_{1}(t)$ of all time point were represented as the 2D matrix with a size of $N_{o s c i} \times N_{t}$. In the third step, to compare the temporal pattern of $V_{1}(t)$ over time, $k$-means clustering across time point was applied for the eigenvector matrices $V_{1}(t)$; as a result, the time samples of $V_{1}(t)$ were classified into $k$-th clusters based on the similarity of the temporal profiles of dFC. Note that the optimal number of clusters was determined using Dunn's method in the LEiDA algorithm (Cabral et al., 2017). Moreover, since we considered the cluster centroids of $V_{1}(t)$ to reflect a recurrent pattern of $V_{1}(t)$ for each cluster, the cluster averaged $d F C(t)$ pattern was reconstructed using the inner product of the centroids of $V_{1}(t)$. In this study, this reconstructed pattern of dFC using centroids of $V_{1}(t)$ for each cluster was defined as the network coupling estimated by the LEiDA algorithm. For more details of this algorithm, see the original article of Cabral et al. (2017). By applying the above-mentioned algorithm proposed by Cabral et al. (2017), the result of estimated temporal changes in phase coupling in the synthetic data was compared with our proposed method.

\subsubsection{Numerical simulation $C$}

To clarify whether our method could sequentially detect the changes in network couplings, we applied our method to 3,000 samples of synthetic time series data of ten coupled oscillators generated by Eq. (15) with the first-order Fourier series. When generating the synthetic data, the noise-scaling factor $p_{i}$ in Eq. (15) was fixed as $p_{i}=0.001$ for each $i$-th oscillator. These synthetic time series consisted of three segments, and the parameter $a_{i j}$ and $b_{i j}$ changed for each segment. The parameter setting for $a_{i j}$ and $b_{i j}$ is shown in Fig. 5. After generating the synthetic data using the above-mentioned procedures, our proposed method was applied to these data, and the network couplings and their change points were evaluated in the same manner as in the prior numerical simulations for the synthetic time series generated with the parameter setting shown in Fig. 5. The precision parameter $\beta$ in Eqs. (9) and (10) was fixed at $\beta^{-1}=0.0001$ for this simulation.

\subsubsection{Validation of our method using EEG data}

Finally, to confirm its neurophysiological validity, we applied our method to real human EEG data obtained by McFadden et al. (2014). This dataset includes EEG recordings from healthy human participants who were passively listening to $40-\mathrm{Hz}$ AM white noise. In general, when listening to 40-Hz AM white noise, $40-\mathrm{Hz}$ oscillatory activity is recorded over the auditory areas (the so-called auditory steady-state response; ASSR (McFadden et al., 2014; Rauschecker \& Scott, 2009; Reyes et al., 2004; Ross et al., 2005)). In addition, a recent ASSR study that used a directional connectivity analysis (Ying et al., 2015) suggested that network connectivity associated with 40-Hz ASSR in healthy humans is mainly distributed at central and fronto-temporal areas. We applied our method to the open EEG dataset to assess whether our method can detect the dynamical changes in neural activity induced by auditory stimuli, as reflected by EEG recordings. 


\section{Segment 1 Segment 2 Segment 3}
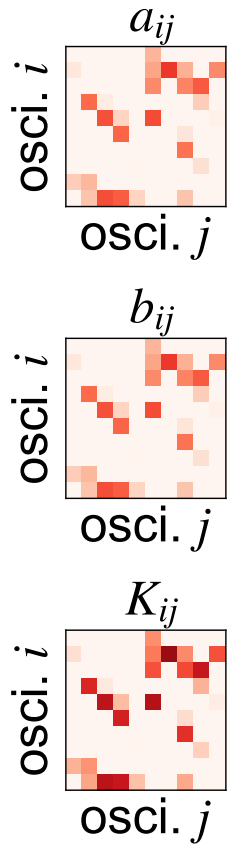
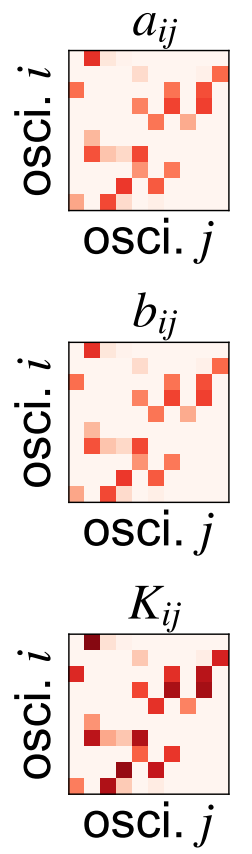
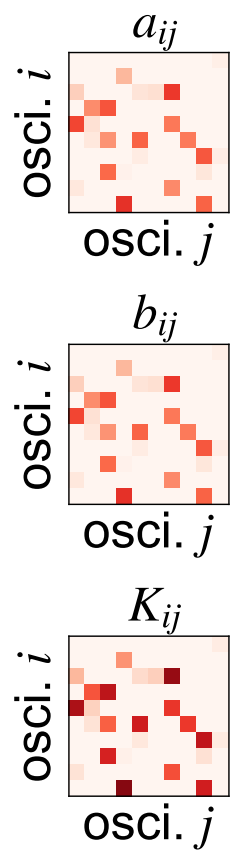

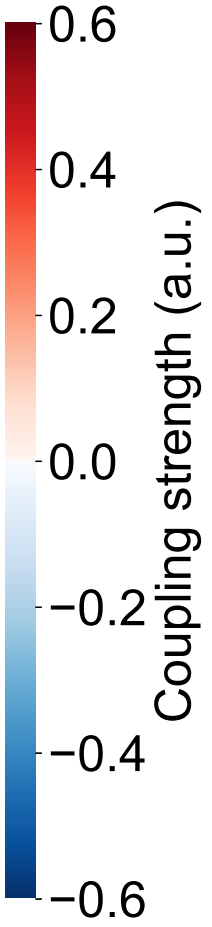

Figure 5: Parameter settings in numerical simulation C. The synthetic data of ten oscillators were generated by the first-order Fourier series-approximated phase-coupled oscillator model with these parameter settings. The generated synthetic time series consisted of three segments separated by two events.

\subsubsection{Open EEG dataset.}

In the dataset used, EEGs were recorded with a 10-10 system-based 64-channel system (Neuroscan, Inc., North Carolina, USA; sampling frequency: 1,000 Hz) in 19 healthy participants who were passively listening to 40-Hz AM noise (McFadden et al., 2014). The AM noise was binaurally presented through foam insert earphones at $75 \mathrm{~dB}$ SPL for $0.5 \mathrm{~s}$ with an inter-trial interval of $1.0 \mathrm{~s}$ (McFadden et al., 2014). The participants performed over 200 trials of this passive listening task (McFadden et al., 2014). A more detailed description of the task settings is provided in the original paper (McFadden et al., 2014). In the following validation of this study, we only used EEG data recorded during the 40-Hz AM noise in participant ID no. 1. Out of the recorded trials, 225 trials were applied and used for the change point detection analysis described in the following section.

\subsubsection{Preprocessing and change point analysis.}

EEG signals were re-referenced off-line to the average of the signals from left and right earlobe electrodes. Then, to reduce the effects of eye movement and eye blink signals, we applied an artifact removal method with empirical mode decomposition (Flandrin et al., 2004, 2005; Molla et al., 2010). A detailed description of this empirical mode decomposition-based electrooculographic artifact removal method is provided in previous articles (Flandrin et al., 2004, 2005). After removing the artifacts, EEGs were converted to current source density signals (Kayser et al., 2006). To extract phase oscillatory dynamics around $40 \mathrm{~Hz}$, the current source density signals were band-pass filtered between 38 and $44 \mathrm{~Hz}$ with a zero-phase FIR filter and converted to analytic signals using the Hilbert transform. Next, phase angles were extracted from the analytic signals of the current source density signals and divided into $1.7 \mathrm{~s}$ epochs (-1.0 to $0.7 \mathrm{~s}, 0.0 \mathrm{~s}$ : stimulus onset) with a $0.7 \mathrm{~s}$ overlap between neighboring epochs.

After preprocessing, the following change point analyses were individually applied to the time series of EEG phase data for each epoch, in the same manner as in the numerical simulation. In the analyses, we 
used data recorded from 10 channels (F3, F4, C3, Cz, C4, P3, Pz, P4, O1, and O2), which were selected according to the International 10-20 system. The first 1,000 samples of the pre-stimulus period (i.e., $-1.0 \leq t \leq 0.0)$ for each epoch were applied to the prior learning for the model, because the numerical simulations revealed that our method requires 500 to 1,000 samples to ensure accurate estimation with 10 coupled oscillators (see the "Results" section for details).

\subsubsection{Performance evaluation.}

In most cases of change point detection problems, the frequency of anomaly changes was lower relative to the normal state frequency; thus, the number of observed samples in each state (i.e., an anomaly state or not) was generally imbalanced. In addition, such changes emerged suddenly. Thus, to evaluate the accuracy of change point detection, it is common to define a time-window around the true change point to allow for minor delays of change point detection (Gensler \& Sick, 2014; Kovács et al., 2020). Furthermore, the time-frequency analysis of the EEG dataset performed in the original article revealed that the time-frequency representation of EEGs related to the 40-Hz ASSR appeared with a delay of around $0.1 \mathrm{~s}$ (McFadden et al., 2014). Thus, to account for a minor delay in the neuronal response, we defined the "error tolerance area" as $0.1 \mathrm{~s}$ after the true time-point of the stimulus onset/offset, as shown in Fig. 6A. The time points corresponding to the change point scores higher than or equal to the pre-defined threshold $\zeta$ were labeled as positive, and all other time points were labeled as negative. To evaluate whether these labels were correct or not, the labels were categorized as shown in Fig. 6B-E. If positive labels were within the error tolerance area, the samples from the first positive point to the end of error tolerance area were tagged as True Positive (Fig. 6B). By contrast, if no positive label was observed within the error tolerance area, all of the time-points corresponding to this area were tagged as False Negative (Fig. 6E). For time-points outside the error tolerance area, time-points with no detection were tagged as True Negative (Fig. 6C), and time points with detection were tagged as False Positive (Fig. 6 D).

However, the accuracy of the change point detection depends on the predefined threshold $\zeta$. Therefore, to evaluate detecting accuracy while considering the effect of the threshold $\zeta$, we analyzed the receiver operating characteristic (ROC) curve and the area under the ROC curve (AUC) was used as the accuracy metric of the change point detection. Thus, the value of the threshold $\zeta$ was changed from 0 to mean $\left(\mathrm{KL}_{\text {base }}\right)+3 \mathrm{SD}\left(\mathrm{KL}_{\text {base }}\right)$, and the true positive rate (TPR) and the false positive rate (FPR) were calculated for each value of threshold $\zeta$ to plot the ROC curve on the graph (Liu et al., 2013). Based on the ROC curve, the AUC value was calculated as the accuracy of change point detection. The ROC curve and AUC were calculated for each epoch individually. Note that the mean $\left(\mathrm{KL}_{\text {base }}\right)$ and $\mathrm{SD}\left(\mathrm{KL}_{\text {base }}\right)$ indicate the mean and standard deviation of the KL divergence (i.e., change point score) in baseline intervals. The baseline was defined as the immediate pre-stimulus period, which was $0.2 \mathrm{~s}$ before stimulus onset. The TPR and FPR were defined as follows:

$$
\begin{aligned}
& T P R=\frac{T P}{T P+F N}, \\
& F P R=\frac{F P}{T N+F P} .
\end{aligned}
$$

By applying the aforementioned evaluation metrics, detection performance was evaluated from 0.0 to $0.6 \mathrm{~s}$ intervals for each epoch. More specifically, we excluded intervals from which data were used for the prior-learning of models and to calculate the threshold of the change point score.

To evaluate the statistical significance of the detection accuracy, the median values of each index over all trials were calculated, and the significance of these values was assessed using a one-tailed permutation test. The null distribution for this permutation test was generated using the following steps:

1. The significance of the change point score of each time sample was assessed based on the threshold $\zeta$. If the score was greater than or equal to the $\zeta$, the time samples were labeled as positive. Otherwise, the samples were labeled as negative.

2. The labels (positive or negative) were shuffled. 
3. The TPR and FPR were evaluated according to these randomized labels.

4. Steps 1 to 3 were repetitively executed between $0 \leq \zeta \leq$ mean $_{\text {base }}+3 \mathrm{SD}_{\text {base }}$ to create the randomized ROC curve.

5. Steps 1 to 4 were carried out for each trial, and the median of the randomized ROC curves for all trials was calculated.

6. All steps were repetitively executed 1,000 times, and the null distribution of the ROC was generated from those 1,000 randomized median ROC curves.

For this statistical analysis, the median of the original ROC curves of all epochs were compared with the null distribution using the above-mentioned steps. 
bioRxiv preprint doi: https://doi.org/10.1101/2020.10.13.335356; this version posted July 28, 2021. The copyright holder for this preprint (which

was not certified by peer review) is the author/funder, who has granted bioRxiv a license to display the preprint in perpetuity. It is made available under aCC-BY-NC-ND 4.0 International license.

A

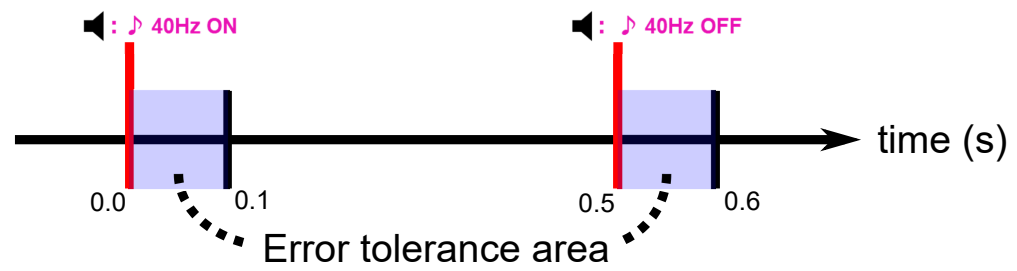

B

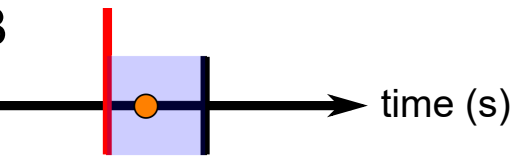

C

True Positive (TP)

True Negative (TN)
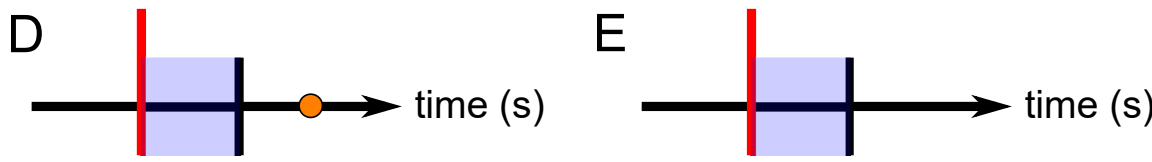

False Positive (FP)

False Negative (FN)

Figure 6: Schematic depiction of the change point evaluation. (A) Schematic depiction of the time protocol of stimulus onset/offset and definition of the error tolerance area. The red vertical lines indicate stimuli onset/offset. The blue shaded areas and the black vertical lines indicate the error tolerance area. (B-E) Relevant situations for the evaluation of the detected change points. If the change point was detected within the error tolerance area, the sample from the first significant point to the end of this area were labeled as True Positive (B). 


\section{Results}

\subsection{Numerical simulation $A$}

To confirm the number of network oscillators and of model learning iterations required to guarantee an accurate estimation of the network couplings when using our proposed method, we first applied the method to the time series of synthetic data with fixed network parameters and compared the estimation result between different numbers of network oscillators.

The results of this simulation are shown in Fig. 7A, which shows the changes in the estimated network couplings for each learning iteration. For less than $N_{\text {osci }}=10$, the estimated structures of the network couplings became close to the exact structure of the synthetic data after 1,000 parameter updating iterations (see iteration 1,000 in Fig. 7A). However, $N_{\text {osci }} \geq 15$ required over 2,000 iterations to accurately estimate the network couplings.

To confirm these results in more detail, we evaluated changes in the estimated error for each learning iteration with all oscillator number conditions (Fig. 7B). As a result, the errors decreased to around zero in conditions with less than $N_{o s c i}=10$ at 1,000 iterations. By contrast, for $N_{\text {osci }} \geq 15$, although the error finally decrease to around zero, the iteration number required to accurately estimate the network couplings gradually increased in proportion to the increase in the number of oscillators.

Furthermore, to confirm the number of learning iterations required to accurately estimate the network couplings, the error for each condition was compared with the $95 \%$ confidence intervals, which were evaluated using surrogate error data for each condition. The results revealed that, when applied for time series data with less than $N_{\text {osci }}=10$, our proposed method can ensure the accurate estimation of network couplings with less than 500 model parameter updating iterations.

Considering these results for numerical simulation A, the practical number of oscillators that could be applied our method to guarantee estimation accuracy is less than $N_{\text {osci }}=10$. Therefore, for the following analyses, we applied our method to the time series data within the number of oscillators (or dimension of time series data) $N_{o s c i}=10$.

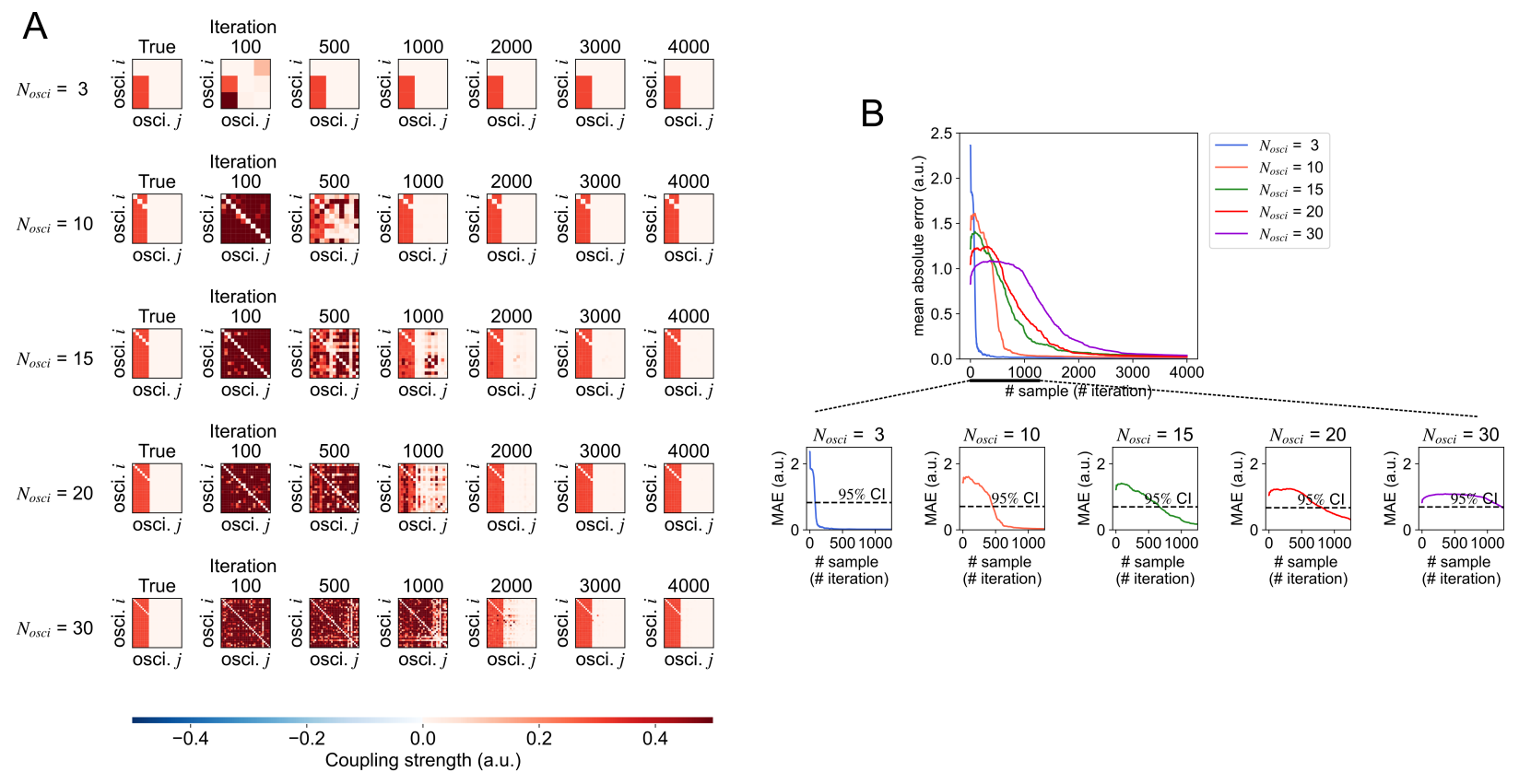

Figure 7: Comparison of true and predicted network structures in numerical simulation A. (A) The changes in the estimated network strength $\hat{\kappa}_{i j}$ for each iteration (note: an iteration indicates number of updates in the model with sequential Bayesian inference). (B) Comparison of changes in the error of network structures for each oscillator number condition. 


\subsection{Numerical simulation $B$}

Considering the results of numerical simulation A, we applied our method to less than ten oscillators in numerical simulation B. We first conducted numerical simulations with synthetic data generated by three phase-coupled oscillator models. The results of this simulation are shown in Figs. 8, 9, 10, and 11.

As shown in Fig. 8, significant increases of the change point scores are located at the boundary between segments 1 and 2 ("Structural change"). Furthermore, significant change point scores were not detected around the boundary between segments 2 and 3 ("Noise-level change"). Thus, we confirmed that the change points were correctly detected only in the case of "Structural change" based on our proposed information theoretical index (i.e., the KL divergence-based index).

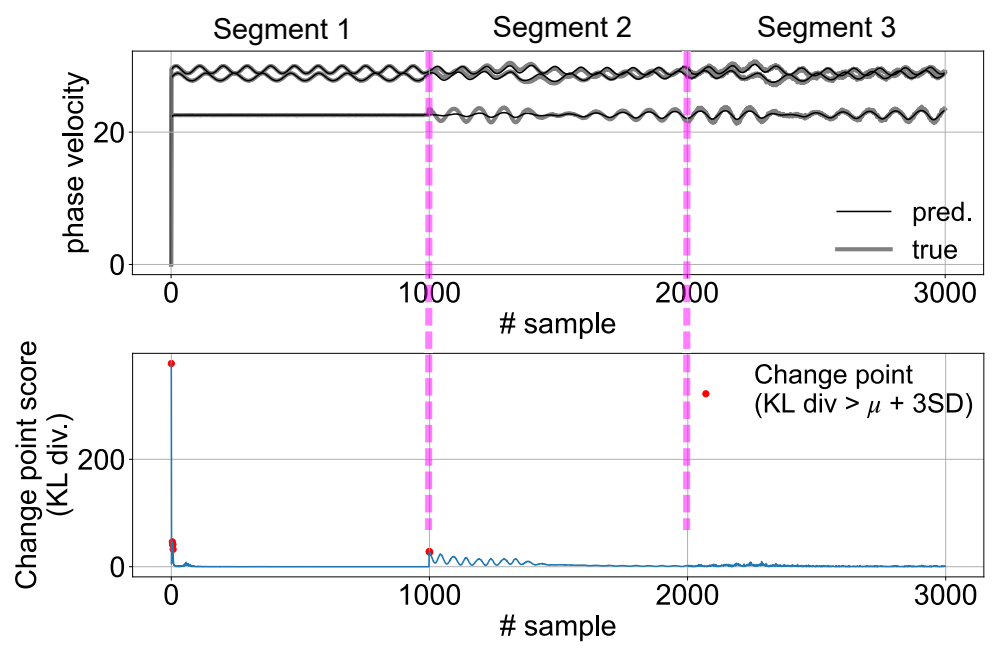

Figure 8: Change point detection in numerical simulation B. The upper panel indicates the time course of the estimated behavior of the phase oscillator model. The black lines and the gray bold lines show the predicted and the true behavior of the phase velocity, respectively. The lower panel indicates the time course of the change point scores. The red dots indicate the selected change points based on the pre-defined threshold. The pink dashed lines show the first and second events (i.e., structural and noise-level change point).

In addition, we attempted to visualize network couplings and phase interaction functions for each segment. Fig. 9 shows the comparison between the true and predicted dynamical structures of the network for each segment. The predicted network couplings shown in Fig. 9B were calculated as the median coupling strengths determined by Eq. (6) with estimated Fourier coefficients for each segment. As shown in Fig. 9, exact and estimated networks using our method were very similar. In particular, our method was able to detect network coupling correctly in segment 1, even though this is where indirect network coupling between the 1st and 2nd oscillators, caused by a common drive from the 3rd oscillator as a confounder, can easily occur. This results emphasize that model-based network analysis is an efficient approach to reduce the false detection of network coupling caused by spurious network couplings.

To further examine whether our proposed method could detect the time-evolving dynamics of oscillatory networks, we compared the predicted function $\Gamma_{i j}\left(\phi_{j}(t)-\phi_{i}(t)\right)$ with the observed interaction for the pair of oscillators for each segment. Fig. 10 shows the median of the functions $\Gamma_{i j}\left(\phi_{j}(t)-\phi_{i}(t)\right)$ for each segment. These functions were evaluated using estimated Fourier coefficients for each segment. If there is no interaction between the $i$ and $j$-th oscillators, the function $\Gamma_{i j}\left(\phi_{j}(t)-\phi_{i}(t)\right)$ will be flat, indicating no interaction between the two oscillators. As shown in Fig. 10A, our model made estimations such that the function $\Gamma_{i j}\left(\phi_{j}(t)-\phi_{i}(t)\right)$ would be flat in the case of no interaction between the pair of oscillators. Moreover, as shown in Fig. 10B and 10C, the predicted functions $\Gamma_{i j}\left(\phi_{j}(t)-\phi_{i}(t)\right)$ were similar between segments 2 and 3, even though the noise-level was quite different between these segments. This indicates that our method can capture changes in the phase interaction function as well as in network coupling. 
bioRxiv preprint doi: https://doi.org/10.1101/2020.10.13.335356; this version posted July 28,2021 . The copyright holder for this preprint (which

was not certified by peer review) is the author/funder, who has granted bioRxiv a license to display the preprint in perpetuity. It is made available under aCC-BY-NC-ND 4.0 International license.

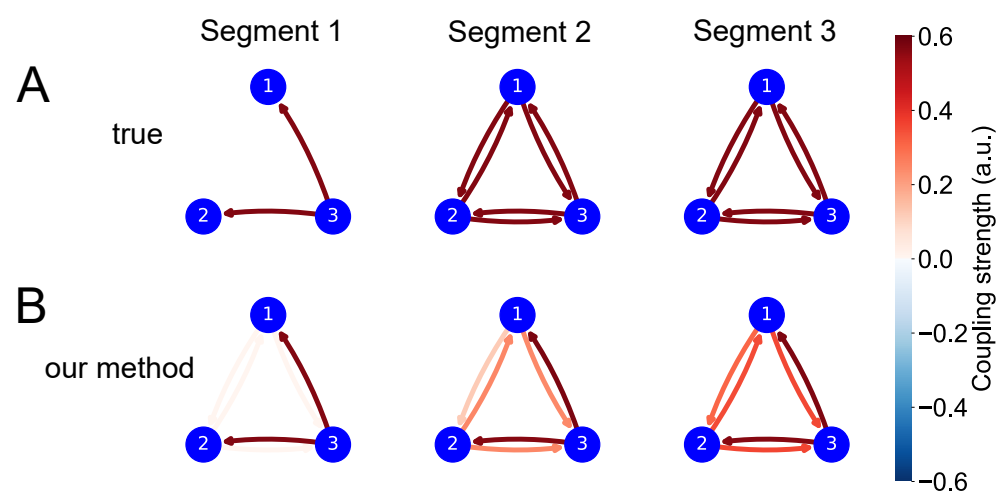

Figure 9: Comparison of true and predicted network structures in numerical simulation B. (A) True network structure in each segment. (B) Predicted network structure based on our proposed method in each segment.

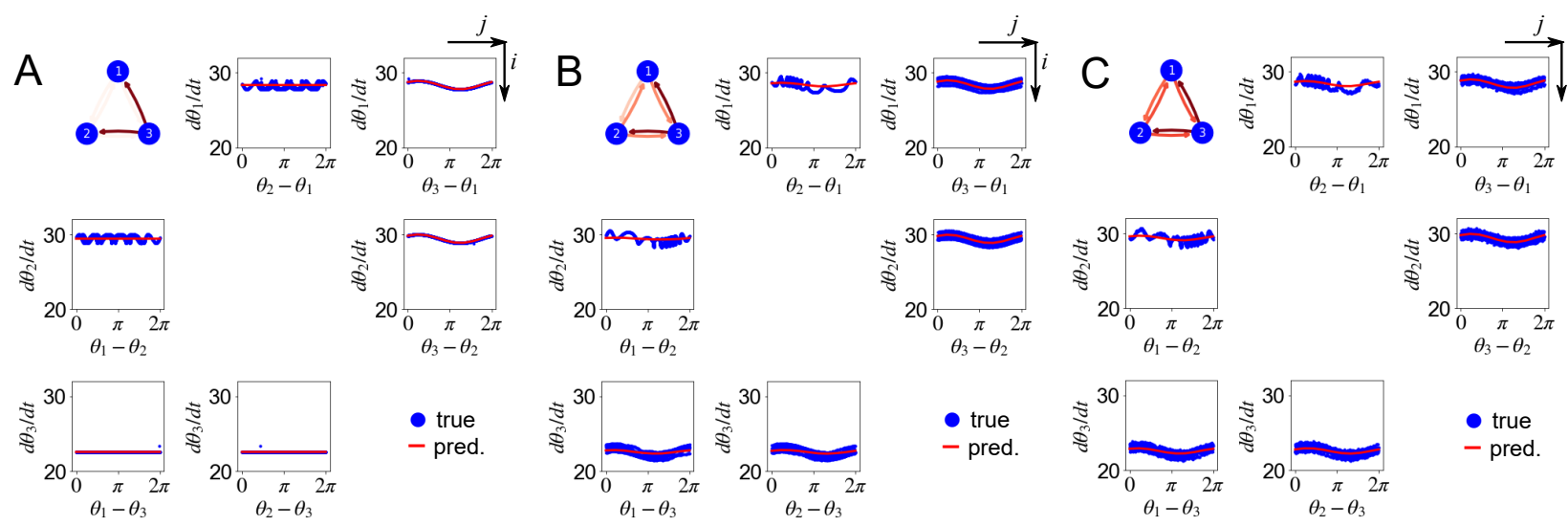

Figure 10: Estimated functions $\Gamma_{i j}\left(\phi_{j}(t)-\phi_{i}(t)\right)$ from synthetic data in numerical simulation B. (A) Result in segment 1. (B) Result in segment 2. (C) Result in segment 3. The upper left diagram in each panel shows the predicted network structure. The blue dots show the actual samples of phase time-series data for all oscillators. The red lines show the averaged phase interaction functions that indicate an interaction from the $j$-th to $i$-th oscillators, estimated by our proposed method in each segment. The phase interaction function would be flat in the absence of an interaction between the $i$ and $j$-th oscillators. 
In addition, to confirm the time course of detection of the network changes estimated by our proposed method, we separated the time samples of synthetic data into distinct intervals under the interpretation that the significant time point of the change point score is a boundary of the interval. Each interval was labeled as State $n$ ( $n$ indicates the number $1,2, \ldots, \mathrm{N}$ ) according to the order of the interval. To compare the detection accuracy of state changes in network coupling, we also analyzed the same data using the conventional clustering-based LEiDA method (Cabral et al., 2017).

Fig. 11 shows the result of this analysis. The lower panel of Fig. 11A shows the exact state label of each time point. Exact network couplings for each state are shown in the upper panel of Fig. 11A. As shown in the lower panel of Fig. 11A, the first 1,000 samples (corresponding to segment 1, mentioned above) are labeled as State 1. Also, the latter 2, 000 samples (corresponding to segments 2 and 3) are labeled as State 2, because the changes in network coupling did not occur between segment 2 and segment 3. Fig. $11 \mathrm{~B}$ and $\mathrm{C}$ show the estimation result of state changes for our method and LEiDA, respectively. As shown in Fig. 11B and C, even though both methods estimated 3 states, the time course of the appearance of each state were quite different depending on the method used. In our method (Fig. 11B), State 1 only appeared in the first several samples. After the first several samples, State 2 appeared until 1,000 samples (see the lower panel of Fig. 11B). As can be seen in the upper panel of Fig. 11B, the network structure of State 2 is very similar to State 1 shown in Fig. 11A. Moreover, the network structure of State 3 in Fig. 11B is not only similar to State 2 in the upper panel of Fig. 11A, but the time course of appearance in both states is also similar. By contrast, as shown in Fig. 11C, quite different network structures were seen for each state estimated by LEiDA, as shown in Fig. 11A. Furthermore, the time course of appearance of each state estimated by LEiDA was transient and randomly changed in whole samples (Fig. 11C).

This result also emphasizes that making the assumption that a dynamical system underlies neural data could be an essential way to consider both temporal dependence and network coupling in such time-evolving dynamics. 
bioRxiv preprint doi: https://doi.org/10.1101/2020.10.13.335356; this version posted July 28, 2021. The copyright holder for this preprint (which was not certified by peer review) is the author/funder, who has granted bioRxiv a license to display the preprint in perpetuity. It is made available under aCC-BY-NC-ND 4.0 International license.

A

true

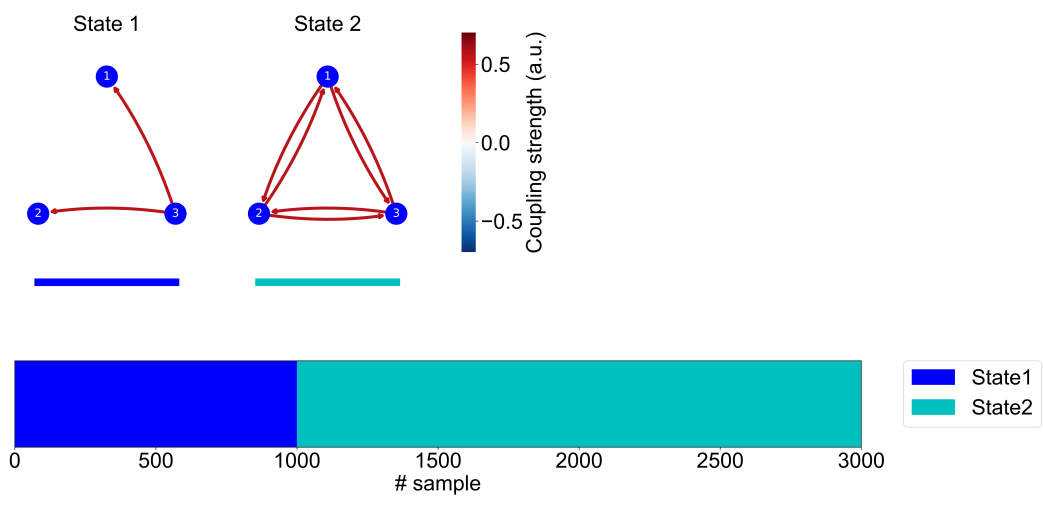

B

\section{our method}

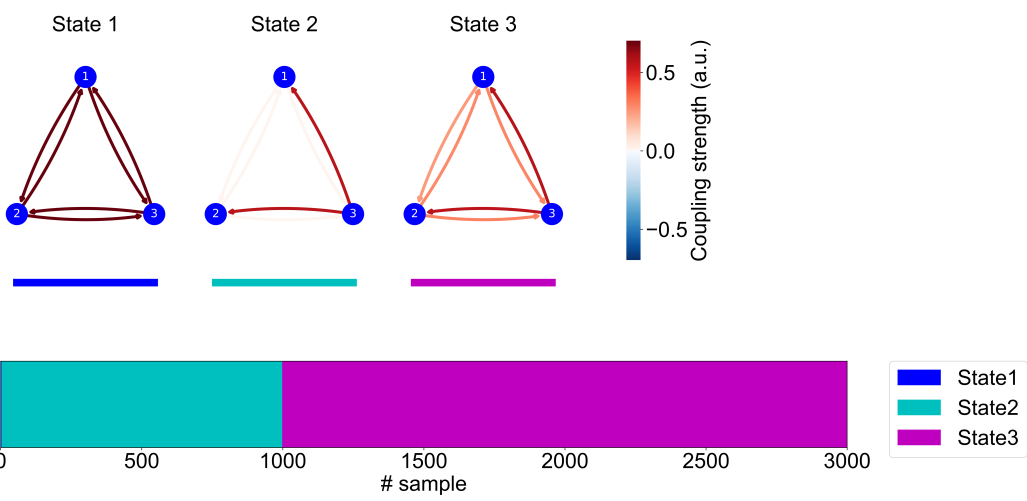

C

\section{LEiDA}
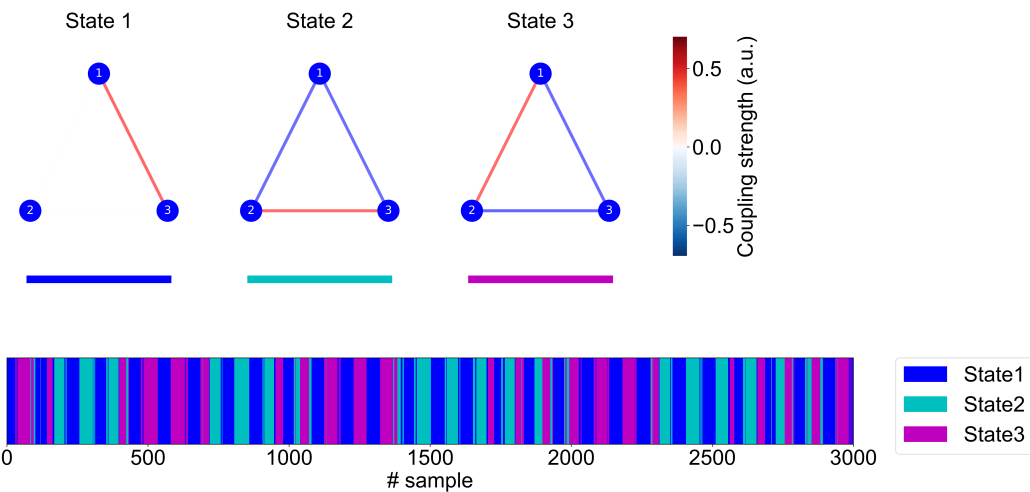

Figure 11: Comparison of the change point estimation results between our method and the previous method (LEiDA). (A) Exact state transition of structural changes in network couplings. The upper panel shows the network coupling of each state. The lower panel indicates the label of state changes for each time sample. (B) The state changes estimated by change point scores using our method. (C) The state changes estimated by the conventional method (LEiDA) proposed by Cabral et al. (2017). 


\subsection{Numerical simulation $C$}

We then applied our method to the synthetic data with ten phase-coupled oscillator models. In this simulation, the synthetic data were generated under the parameter condition as shown in Fig. 5. In this parameter setting, the time series of synthetic data were generated from 3,000 samples, and these samples were divided into three segments by two events. For each segment, the Fourier coefficient parameters corresponding to the network coupling were changed.

The result for this simulation is shown in Fig. 12. As can be seen in Fig. 12B, a significant increase of the change point score was detected around the boundary for each segment (see the red dot in this figure). Moreover, as shown in Fig. 12A, the predicted network couplings of each segment were relatively similar to the true network couplings in the synthetic data for each segment. These results indicated that our method could also be applied for ten coupled oscillators as the detector of the change point of dynamical structure in phase-coupled oscillator networks.

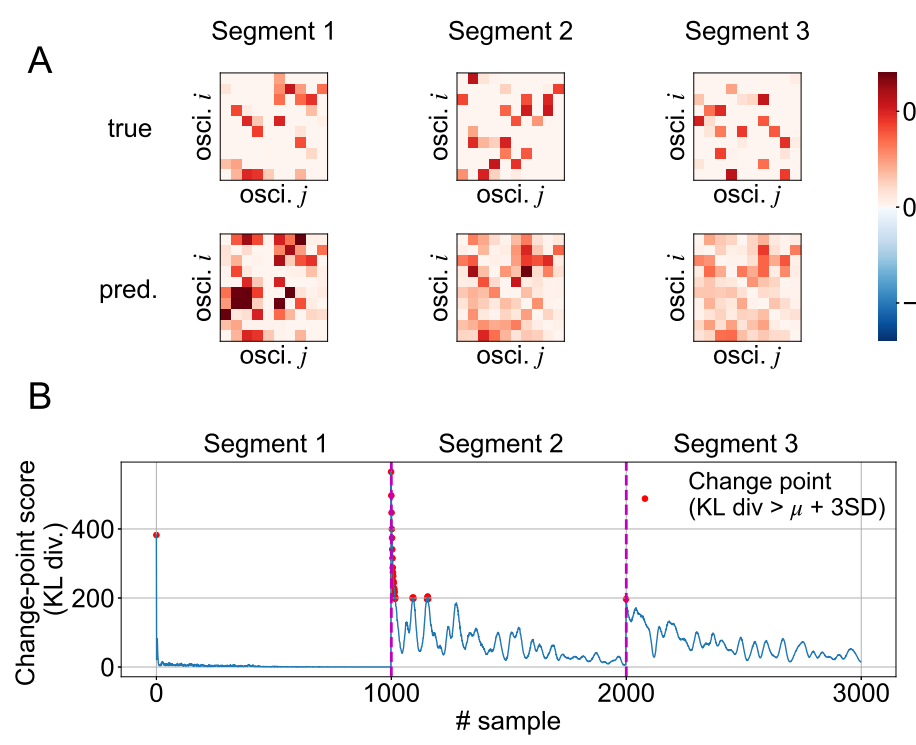

Figure 12: Change point detection in numerical simulation C. (A) Comparison of true and estimated network couplings. The upper three panels show the true network couplings in each segment. The lower three panels show the estimated network couplings in each segment estimated by our method. (B) The time course of the change point scores. The red dots indicate the selected change points based on the pre-defined threshold. The pink dashed lines show exact timing of the changes in network couplings. 


\subsection{Scalp EEG data}

Finally, we evaluated the neurophysiological validity of our method by applying it to EEG data that were shared by McFadden et al. (2014). Figs. 13A and 13B show the typical correct and incorrect results of change point detection in EEG data from participant 1, when the threshold $\zeta$ was set as $\zeta=$ mean $\left(\mathrm{KL}_{\text {base }}\right)$ $+3 \mathrm{SD}\left(\mathrm{KL}_{\text {base }}\right)$. As shown in these figures, the change point scores were significantly increased around the stimulus onset and offset in epochs for which the change point within the error tolerance area was successfully detected, while in epochs for which there was an incorrect detection, the significant increase of the change point scores was outside the error tolerance area.

As mentioned above, the accuracy of the change point detection is dependent on the predefined threshold $\zeta$. Therefore, to consider this issue, we applied the ROC curve analysis to evaluate accuracy. In this analysis, the value of the threshold $\zeta$ was changed from 0 to mean $\left(\mathrm{KL}_{\text {base }}\right)+3 \mathrm{SD}\left(\mathrm{KL}_{\mathrm{base}}\right)$, and the TPR and FRP were calculated for each value of $\zeta$ to plot the ROC curve, as shown in Fig. 13C. As a result, the median of the original ROC curves over 255 trials showed a large AUC score relative to the median of permutation ROC curves. Furthermore, the AUC score, which was calculated according to the

median of the original ROC curves, was 0.8245 . Therefore, although the ability of our method to ensure the validity of change point detection was limited by the number of EEG electrodes, the results indicate that our method successfully detected the changes in EEG phase oscillatory dynamics with significant accuracy. 

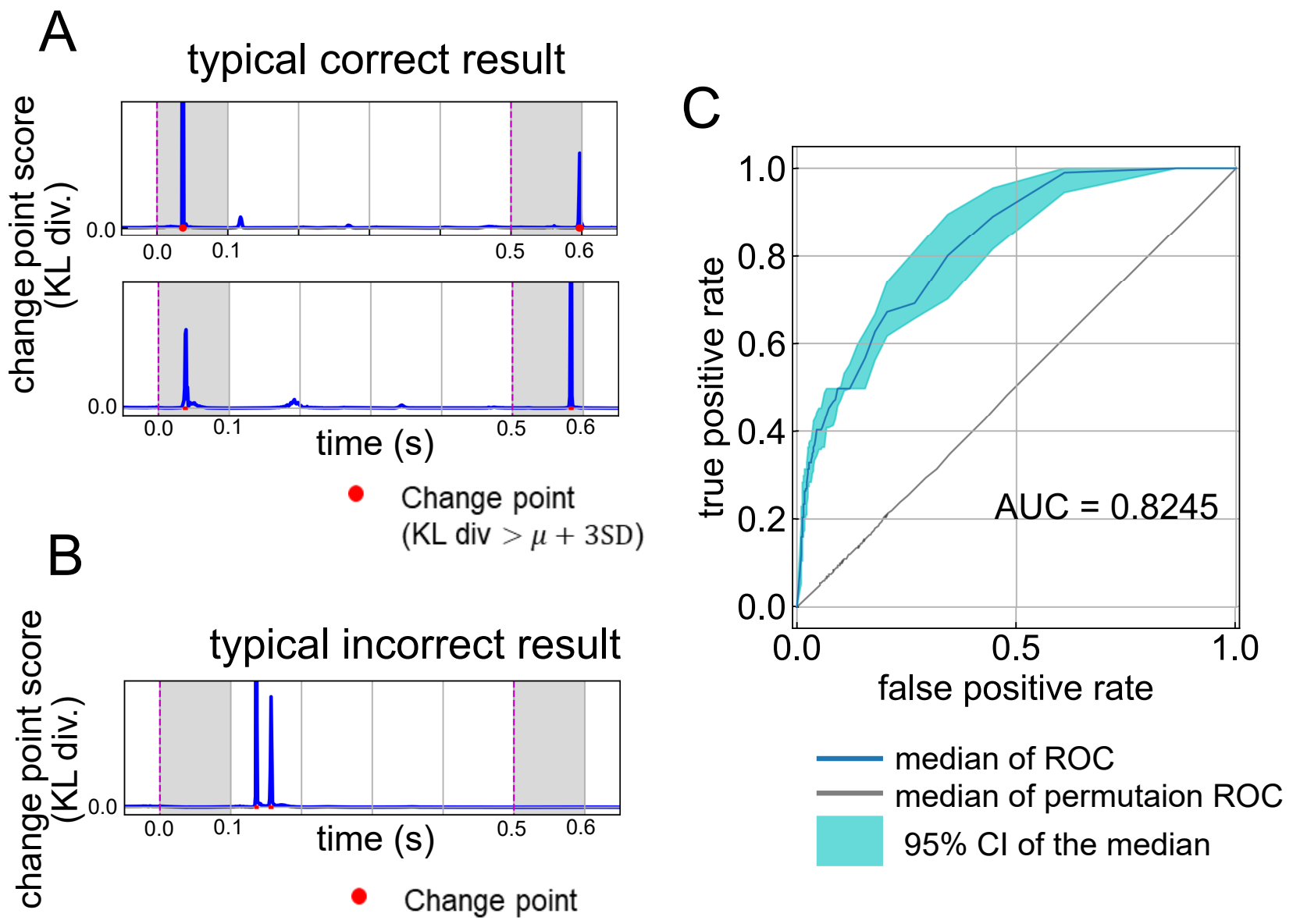

$(\mathrm{KL} \operatorname{div}>\mu+3 \mathrm{SD})$

Figure 13: Change point detection analysis in the EEG dataset. (A, B) Typical examples of correct and incorrect epochs in change point detection in EEG data (when the threshold $\zeta$ is set as $\zeta=\mu+3 S D$ ), respectively. (C) The median ROC curve of all 255 epochs. Blue lines and the light blue shaded area indicate the median ROC curve and $95 \%$ confidence intervals (CI) of the median of ROC curves. Gray lines indicate the permutation median of the ROC curves. The ROC curve for each epoch was drawn by plotting the data points of the false positive rate and true positive rate for each threshold $\zeta$ (from 0 to $\mu+3 S D$ ). 


\section{Discussion}

In this study, we proposed a new approach to detect the change points of brain networks by extending the data-driven method to estimate phase oscillatory dynamics based on sequential Bayesian inference. To validate our proposed method, we applied it to numerical and empirical neurophysiological data. The numerical data analyses confirmed that the change point score of our method significantly increases only when structural changes in the dynamical network occur, and not when noise-level changes in the dynamical network occur. These results support the two following assumptions for our proposed method: (1) time-evolving dynamics and data structures of functional brain networks can be represented by a phase-coupled oscillator model, and (2) temporal changes in dynamical brain structures are reflected in changes in model parameters. Furthermore, the neurophysiological data analysis revealed that our method was able to detect the change point of phase oscillatory dynamics induced by auditory stimuli with an $A U C=0.8245$ accuracy, which was based on the median of original ROC curves for all epochs. These findings suggest that our proposed method has mathematical and neurophysiological validity. In the following section, we will discuss the neuroscientific significance of our proposed method, and will compare our results with those of previous studies that have employed time-varying functional connectivity analyses.

\subsection{Advantages of our proposed method}

The originality of our proposed method lies in the application of sequential Bayesian inference to estimate the phase interaction function of dynamical networks and to detect the change point of such dynamics. Using this approach, the current state of network dynamics of the brain could be expressed as a probability distribution (Figs. 1 and 2). Therefore, our method is not only able to visualize time-varying network couplings of the brain, but can also represent the extent of the time-varying changes in dynamical brain structures using an information theoretical index (the KL divergence).

Relevant previous studies have reported three typical methods that can be used to estimate temporal changes in the network connectivity of neural activity. First, relevant conventional studies include dFC studies to estimate time-varying changes in functional connectivity of the human brain (Hutchison et al., 2013; Damaraju et al., 2014). This method extends the functional connectivity analysis to estimate time-varying network structures by applying the sliding window method (Hutchison et al., 2013; Damaraju et al., 2014). Although dFC analysis focuses on the temporal changes in brain networks, this method separately evaluates functional networks with static indices, such as the correlation between time-series data, for each time window. Thus, these analyses do not explicitly consider the time-evolving dynamics of time-series data, because multivariate time series with non-stationary dynamics usually contain temporal dependence and auto-correlation, as in a dynamical system. Moreover, in most of the studies using dFC, when evaluating the network structure in each time window, the coupling strength for each pair of brain areas is calculated based on the temporal correlation with the time-series data. According to prior evidence (Granger et al., 1974), correlation coefficients between two different time-series data tend to be large, regardless of the existence of causality between two time-series data. Therefore, this method could lead easily to the detection of incorrect network connectivity based on spurious correlations. The second method is a clustering-based algorithm to estimate the phase oscillatory network couplings, named LEiDA (Cabral et al., 2017), which is mentioned in numerical simulation B. In this method, the strength of coupling is calculated based on the pairwise phase coherence of two different time-series. Although the LEiDA does not apply a sliding window, time-evolving dynamics would not be considered, because state transition estimates are based on the $k$-mean clustering over samples. In other words, the LEiDA separates the time-series into $k$ segments without considering the temporal dependence (i.e., the causal relationship from the past to current states in the time-series data). Therefore, this algorithm cannot strictly consider the time-evolving dynamics of network interactions in whole brain regions. As shown by our numerical simulation results (see Fig. $11 \mathrm{~A}$ and $\mathrm{C}$ ), the LEiDA results had an incorrect network estimation and incorrect temporal transition. Thus, making the assumption that a dynamical system underlies neural data could be an essential way to consider both temporal dependence and network coupling in such time-evolving dynamics. The third method is the change point detection algorithm of the time-varying gene regulatory networks by combining the auto-regressive (AR) model and Kalman filtering (Xiong \& Zhou, 2013). The Kalman filter is based on 
the state-space model framework with a Bayesian parameter updating approach. Xiong \& Zhou (2013) combined the recursive parameter estimation of the AR model and Kalman filtering by giving the model parameters of the AR model for the state variables (i.e., model parameters in the AR model are estimated as state variables using Kalman filtering). Unlike the two prior mentioned methods (dFC and LEiDA), this method is more advanced in its ability to estimate time-varying network connectivity by considering the time-evolving dynamics based on the state-space model. Moreover, this method is similar to our own method, in that it also applies a Bayesian approach to estimate the model parameters. However, Xiong \& Zhou (2013) adopted the AR model for the time-evolving dynamics of time series in gene regulatory networks. Namely, this method explicitly assumes that both the network interaction and temporal dynamics in the time series data are linear. In the case of synchronous neural oscillatory data, time-evolving dynamics driven by the interactions for each oscillator could be considered as a coupled nonlinear system. Considering the possibility that a nonlinear dynamical system, such as a phase-coupled oscillator, underlies neural data might be important to estimate the time-varying network coupling for neural data.

Our proposed method advances the above conventional methods (Hutchison et al., 2013; Damaraju et al., 2014; Cabral et al., 2017) in the two following ways. First, our method considers both time-evolving dynamics and a nonlinear dynamical structure of functional brain networks. Unlike the dFC and LEiDA frameworks, our method adequately considers nonlinear and time-evolving properties of the dynamical brain activity by applying a model-based approach to estimate functional brain networks. The advantage of such a model-based approach lies in the fact that misinterpretation caused by spurious correlations is largely reduced by applying a network system with model-based temporal dynamics. As revealed by numerical simulation B (Figs. 9 and 11), our method was able to correctly detect network coupling in this segment, even in situations where the LEiDA algorithm led to incorrect estimations. Second, our method can sequentially detect changes in dynamical brain structures based on Bayesian inference and an information-theoretic metric (i.e., KL divergence). Moreover, our method can consequently consider the temporal dynamics of time-series data by applying sequential Bayesian inference to estimate the time-varying changes in the model parameters. As a subsidiary advantage of applying the phase-coupled oscillator model to estimate network dynamics of the brain, directional network couplings could be detected, which is not the case with dFC and LEiDA analyses.

In summary, by adopting the property of time-evolving dynamics in the observed data using a dynamical model, the proposed method succeeded in providing a quantitative interpretation of the change point of time-evolving dynamics in brain networks using probabilistic inference and an information-theoretic metric.

\subsection{Neuroscientific interpretation and generalizability}

In this study, by supposing that the phase-coupled oscillator model is a representation of the time-evolving dynamics of functional brain networks, we expected the changes of functional brain networks to be quantified as changes in dynamical structures of the model parameters. Based on this assumption, we applied our proposed method to a multi-channel EEG time series that was measured from a healthy participant who was passively listening to 40-Hz AM noise (McFadden et al., 2013, 2014). In general, when listening to 40-Hz AM noise, EEG signals emanating from a broad area of the brain are synchronized at around 40 Hz. (McFadden et al., 2014; Rauschecker \& Scott, 2009; Reyes et al., 2004; Ross et al., 2005). Our method could successfully detect changes in dynamical structures when changes in dynamical structures of the network occurred with regards to the ASSR. This indicates that a model-based approach to detect the change point of functional brain networks is more convenient to interpret the temporal dynamics of the brain. Moreover, another remarkable point is that our method can detect changes in the dynamical structures reflected in scalp EEGs on a single-trial basis.

In summary, our proposed method successfully detected the dynamical structures of the brain networks, as well as the change points of those dynamical structures. Considering the increasing interest of neuroscientists in the relationship between flexible changes in functional brain networks and cognitive functions (Hutchison et al., 2013; Bassett et al., 2011; Baum et al., 2017; Braun et al., 2015, 2016), our method could help reveal the crucial role of flexible changes in dynamical structures of the brain. 


\subsection{Significance of the proposed method in the context of change point detection}

The change point is defined according to the model parameter $K$ regarding the probability density $p_{K}(x)$ of the time series $x$ changes from $K=K_{0}$ to $K=K_{1}$ (i.e., $K_{0} \neq K_{1}$ ), and the task of detecting the timing of such changes is called the change point problem. Change point detection has been actively studied in machine learning and data mining research over the past few decades (Basseville et al., 1993). Most prior methods of change point detection have been developed according to the common framework that the extent of abrupt changes in the time series data is measured using the log-likelihood ratio to compare the past and the current state of the probability density (Basseville et al., 1993). For the typical approach based on the log-likelihood ratio, the following methods can be considered: the cumulative sum of log-likelihood ratio algorithm (Basseville et al., 1993; Gustafsson \& Gustafsson, 2000), the generalized log-likelihood ratio (Basseville, 1988; Gustafsson, 1996; Gustafsson \& Gustafsson, 2000), and the AR model-based approach (Basseville, 1988; Yamanishi \& Takeuchi, 2002). However, these typical methods are specialized for a single sequence, and a method that focuses on the time-evolving dynamics of network connectivity is still lacking. In a recent study, Idé and colleagues (Idé et al., 2009; Idé, 2014) proposed an approach for detecting structural changes in the network sequence based on the sparse Gaussian graphical model (graphical lasso), but this method estimates the graph structure assuming that there is a linear correlation between each node in the network. Therefore, this method is difficult to apply for multivariate time series data in which there is an underlying nonlinear dynamical system, such as synchronous neural activity data. As mentioned in the previous discussion (see section 4.1), in the field of bioinformatics, Xiong \& Zhou (2013) proposed a Kalman filter-based change point detection algorithm for the time-varying gene regulatory network. However, this algorithm also assumes a linear model for temporal dynamics in the network.

Comparing our proposed method with those of the above-mentioned prior studies, our method also has the limitation that it applies a parametric model to estimate the network structure; however, our method has the advantage in that it can simultaneously estimate both visualization of the network structure and detection of temporal changes in such a network. Applying sequential Bayesian inference to estimate the model parameter is a common approach to model fitting. However, our method is unique in measuring the extent of the changes in model parameters by comparing prior and posterior distributions of model parameters for quantification of the change point score.

\subsection{Limitations of this study}

Despite the advantages of our proposed method, some limitations exist. First, the expressive power of phase dynamics in our method is strongly dependent on the pre-defined function of the dynamical model. Since phase dynamics are approximated by the Fourier series in our method, prediction performance and reliability depend on the dimension of the Fourier series. This is one of the reasons why our proposed method could not be applied for time series data with over $N_{\text {osci }}=10$. Second, the detection accuracy is also affected by the precision factor $\beta$, which was fixed at $\beta^{-1}=0.0001$ in this study. As can be seen in Eq. (10), the parameter $\beta$ affects the observed noise in the model. Given that $\boldsymbol{f} \boldsymbol{\Sigma}_{\boldsymbol{K}} \boldsymbol{f}^{T}$, which is the first term of the right-hand side of Eq. (10), tends to be around zero throughout the sequential update of the predictive distributions, the observation noise of the model converges with $\beta^{-1} I$. This tendency directly influences the variance of the fitting error of the model. Thus, both the dimension of the Fourier series and $\beta$ affect the performance of change point detection. However, these parameters should be selected exploratory, because the optimal setting for each parameter depends on the complexity and dimensionality of the data. The optimal dimension of the Fourier series $P$ could be estimated using the Akaike information criterion or Bayesian information criterion. For simplification, in this study, even though the dimension of the Fourier series is fixed at $P=1$, future work should consider the dimensionality using the above-mentioned information criteria when applying the extended version of the proposed method. Moreover, to more strictly consider observation noise of the data, applying a variational Bayesian linear regression approach with the Gamma prior distribution for the observation noise covariance (Tzikas et al., 2008) would enable us to deal with this issue. However, further study is required to attempt this approach.

An additional limitation of our method is the number of oscillators required to guarantee an accurate estimation of the network couplings. As shown in Fig. 7, the practical number of oscillators that could be 
accurately estimated by the proposed method is less than $N_{\text {osci }}=10$. The above-mentioned conventional methods to estimate the time-varying functional connectivity, such as dFC and LEiDA, have no such limitation; however, these methods could lead to the incorrect detection of network couplings, as mentioned above.

Despite these limitations, our method provides advantages over the conventional methods in that both visualization of the network structure and detection of the change point are realized in parallel using Bayesian inference.

\section{Conclusions}

In this study, we tested a new method for detecting changes in dynamical structures of the brain. By applying our proposed method to numerical and electroencephalographic data, we confirmed that our method can successfully detect the change points at which dynamical changes of the network occur. These results suggest that our proposed method could help reveal the neural basis of dynamical changes of brain networks. Furthermore, although it is beyond the scope of the current study, our proposed method would be useful to examine more details of the cognitive functions and learning capacity, which are reflected in the time-evolving dynamics of brain networks. To apply our proposed method in future neuroscience studies, future studies are required to address some of the limitations mentioned above.

\section{Acknowledgements}

This research was funded by the JSPS KAKENHI grant (20K19867, https://kaken.nii.ac.jp/en/grant/KAKENHI-PROJECT-20K19867/) from the Japan Society for the Promotion of Science.

\section{Credit author statement}

Conceptualization, H.Y. and K.K.; Methodology, H.Y.; Software, H.Y.; Validation, H.Y. and K.K.; Formal analysis, H.Y.; Investigation, H.Y.; Resources, H.Y. and K.K.; Data curation, H.Y.; Writing-original draft preparation, H.Y.; Writing-review and editing, H.Y. and K.K.; Visualization, H.Y.; Supervision, K.K.; Project administration, K.K.; Funding acquisition, H.Y. All authors have read and agreed to the published version of the manuscript.

\section{Data and code availability statements}

Data will be made available to all interested researchers upon request. The sample code of the numerical simulation described in this paper is available at Github: https://github.com/myGit-YokoyamaHiroshi/ChangeDetectSim

\section{Appendix A. Dynamical systems theory of the phase oscillator model}

As we mentioned in section 2.1, from the point of view of dynamical systems, the time series $\boldsymbol{X} \in \mathbb{R}^{\mathrm{N}}$, which is followed by a periodic function $F$, can be described as follows:

$$
\frac{d \boldsymbol{X}}{d t}=F(\boldsymbol{X})
$$

If the system exhibits limit cycles with stable point $\boldsymbol{X}=\boldsymbol{X}_{\tau}(\boldsymbol{\phi})$, the polar coordinates of this system can be described as follows:

$$
\frac{d \boldsymbol{\phi}}{d t}=\left.\operatorname{grad}_{\boldsymbol{X}} \phi\right|_{\boldsymbol{X}=\boldsymbol{X}_{\tau}(\boldsymbol{\phi})} \cdot F\left(\boldsymbol{X}_{\tau}(\boldsymbol{\phi})\right)=\boldsymbol{\omega}
$$


where $\boldsymbol{\phi}, \boldsymbol{\omega}$, and $\tau$ denote the phase, natural frequency, and one-cycle period, respectively. Phase dynamics follow the periodic cycle, which is described as $\phi(t+\tau)=\phi(t)$, around the stable point.

Here, we consider two coupled oscillators, $\boldsymbol{X}=\left\{x_{i}, x_{j}\right\}$, driven by an interaction between oscillators. In this case, the dynamics can be described as follows:

$$
\frac{d x_{i}}{d t}=F_{i}\left(x_{i}\right)+g_{i j}\left(x_{j}, x_{i}\right),
$$

where $F_{i}\left(x_{i}\right)$ and $g_{i j}\left(x_{j}, x_{i}\right)$ indicate the dynamical system function of the $i$-th oscillator and the interaction between $i$ and $j$-th oscillators, respectively. In the polar coordinates, Eq. (A.3) can be expanded around the stable point $X_{\tau}(\Phi)=\left\{x_{\tau}\left(\phi_{i}\right), x_{\tau}\left(\phi_{j}\right)\right\}$, as described in the following equation:

$$
\begin{aligned}
\frac{d \phi_{i}}{d t} & =\left.\operatorname{grad}_{\boldsymbol{X}} \phi\right|_{\boldsymbol{X}=x_{\tau}\left(\phi_{i}\right)} \cdot\left\{F\left(x_{\tau}\left(\phi_{i}\right)\right)+g_{i j}\left(x_{\tau}\left(\phi_{i}\right), x_{\tau}\left(\phi_{j}\right)\right)\right\} \\
& =\omega+Z\left(\phi_{i}\right) \cdot g_{i j}\left(x_{\tau}\left(\phi_{i}\right), x_{\tau}\left(\phi_{j}\right)\right),
\end{aligned}
$$

where $Z\left(\phi_{i}\right)=\left.\operatorname{grad}_{\mathrm{X}} \phi\right|_{\mathrm{X}=x_{\tau}\left(\phi_{i}\right)}$ indicates the function of the phase-response curve corresponding to the $i$-th oscillator.

By introducing relative phase $\psi_{i}$ via $\phi_{i}=\omega t+\psi_{i}$, this can be rewritten as follows:

$$
\frac{d \psi_{i}}{d t}=Z\left(\omega t+\psi_{i}\right) \cdot g_{i j}\left(x_{\tau}\left(\omega t+\psi_{i}\right), x_{\tau}\left(\omega t+\psi_{j}\right)\right) .
$$

Moreover, by averaging over one cycle with the period $T$, this can be rewritten as follows:

$$
\frac{d \psi_{i}}{d t}=\frac{1}{T} \int_{0}^{T} Z\left(\omega t+\psi_{i}\right) \cdot g_{i j}\left(x_{\tau}\left(\omega t+\psi_{i}\right), x_{\tau}\left(\omega t+\psi_{j}\right)\right) d t .
$$

If $\omega t=\theta$ is substituted to obtain $d t=\frac{1}{\omega} d \theta$, then $d t=\frac{T}{2 \pi} d \theta$. Therefore, we obtain the following equation:

$$
\frac{d \psi_{i}}{d t}=\frac{1}{2 \pi} \int_{0}^{2 \pi} Z\left(\theta+\psi_{i}\right) \cdot g_{i j}\left(x_{\tau}\left(\theta+\psi_{i}\right), x_{\tau}\left(\theta+\psi_{j}\right)\right) d \theta
$$

In addition, assuming that $\Theta=\theta+\psi_{i}$ (i.e., $\theta=\Theta-\psi_{i}$ ), Eq. (A.5) can be approximated as the function of phase difference $\psi_{j}-\psi_{i}$ (Kuramoto, 1984; Pietras \& Daffertshofer, 2019), described as follows:

$$
\begin{aligned}
\frac{d \psi_{i}}{d t} & =\Gamma_{i j}\left(\psi_{j}-\psi_{i}\right) \\
\Gamma_{i j}\left(\psi_{j}-\psi_{i}\right) & =\frac{1}{2 \pi} \int_{0}^{2 \pi} Z(\Theta) \cdot g_{i j}\left(x_{\tau}(\Theta), x_{\tau}\left(\Theta+\left(\psi_{j}-\psi_{i}\right)\right)\right) d \Theta
\end{aligned}
$$

Note that the function $\Gamma_{i j}\left(\psi_{j}-\psi_{i}\right)$ describes the interaction between the $i$ and $j$-th oscillators.

Considering Equations (A.5)- (A.9), Eq. (A.4) can be extended as the function of phase difference between oscillators (Kuramoto, 1984; Pietras \& Daffertshofer, 2019), as follows:

$$
\frac{d \phi_{i}}{d t} \approx \omega+\Gamma_{i j}\left(\phi_{j}-\phi_{i}\right)
$$

Given the above equations, the function $\Gamma_{i j}\left(\phi_{j}-\phi_{i}\right)$ describes the phase changes in the cycle period of coupled oscillators via network interactions for each oscillator. Thus, by estimating the function $\Gamma_{i j}\left(\phi_{j}-\phi_{i}\right)$ from empirically measured data, we can characterize the interaction between oscillators. 


\section{References}

Bassett, D. S., Wymbs, N. F., Porter, M. A., Mucha, P. J., Carlson, J. M., \& Grafton, S. T. (2011). Dynamic reconfiguration of human brain networks during learning. Proceedings of the National Academy of Sciences of the United States of America, 108, 7641-7646. doi:10.1073/pnas.1018985108.

Basseville, M. (1988). Detecting changes in signals and systems-A survey. Automatica, 24, 309-326. URL: https://linkinghub.elsevier.com/retrieve/pii/0005109888900738. doi:10.1016/0005-1098(88)90073-8.

Basseville, M., Nikiforov, I. V. et al. (1993). Detection of abrupt changes: theory and application volume 104. Prentice hall Englewood Cliffs.

Baum, G. L., Ciric, R., Roalf, D. R., Betzel, R. F., Moore, T. M., Shinohara, R. T., Kahn, A. E., Vandekar, S. N., Rupert, P. E., Quarmley, M., Cook, P. A., Elliott, M. A., Ruparel, K., Gur, R. E., Gur, R. C., Bassett, D. S., \& Satterthwaite, T. D. (2017). Modular Segregation of structural brain networks supports the development of executive function in youth. Current Biology, 27, 1561-1572.e8. URL: http://dx.doi.org/10.1016/j.cub.2017.04.051. doi:10.1016/j.cub.2017.04.051.

Bishop, C. M. (2007). Pattern recognition and machine learning (Information science and statistics). (1st ed.). Springer.

Braun, U., Schäfer, A., Bassett, D. S., Rausch, F., Schweiger, J. I., Bilek, E., Erk, S., Romanczuk-Seiferth, N., Grimm, O., Geiger, L. S., Haddad, L., Otto, K., Mohnke, S., Heinz, A., Zink, M., Walter, H., Schwarz, E., Meyer-Lindenberg, A., \& Tost, H. (2016). Dynamic brain network reconfiguration as a potential schizophrenia genetic risk mechanism modulated by NMDA receptor function. Proceedings of the National Academy of Sciences of the United States of America, 113, 12568-12573. doi:10.1073/pnas. 1608819113.

Braun, U., Schäfer, A., Walter, H., Erk, S., Romanczuk-Seiferth, N., Haddad, L., Schweiger, J. I., Grimm, O., Heinz, A., Tost, H., Meyer-Lindenberg, A., \& Bassett, D. S. (2015). Dynamic reconfiguration of frontal brain networks during executive cognition in humans. Proceedings of the National Academy of Sciences of the United States of America, 112, 11678-11683. doi:10.1073/pnas.1422487112.

Breakspear, M., Heitmann, S., \& Daffertshofer, A. (2010). Generative models of cortical oscillations: neurobiological implications of the kuramoto model. Frontiers in Human Neuroscience, 4, 1-14. URL: http://journal.frontiersin.org/article/10.3389/fnhum.2010.00190/abstract. doi:10.3389/fnhum.2010.00190.

Cabral, J., Hugues, E., Sporns, O., \& Deco, G. (2011). Role of local network oscillations in resting-state functional connectivity. NeuroImage, 57, 130-139. URL: http://dx.doi.org/10.1016/j.neuroimage.2011.04.010. doi:10.1016/j.neuroimage.2011.04.010.

Cabral, J., Vidaurre, D., Marques, P., Magalhães, R., Silva Moreira, P., Miguel Soares, J., Deco, G., Sousa, N., \& Kringelbach, M. L. (2017). Cognitive performance in healthy older adults relates to spontaneous switching between states of functional connectivity during rest. Scientific Reports, 7, 1-13. doi:10.1038/s41598-017-05425-7.

Damaraju, E., Allen, E. A., Belger, A., Ford, J. M., McEwen, S., Mathalon, D. H., Mueller, B. A., Pearlson, G. D., Potkin, S. G., Preda, A., Turner, J. A., Vaidya, J. G., Van Erp, T. G., \& Calhoun, V. D. (2014). Dynamic functional connectivity analysis reveals transient states of dysconnectivity in schizophrenia. NeuroImage: Clinical, 5, 298-308. URL: http://dx.doi.org/10.1016/j.nicl.2014.07.003. doi:10.1016/j.nicl.2014.07.003.

Deco, G., Cabral, J., Woolrich, M. W., Stevner, A. B., van Hartevelt, T. J., \& Kringelbach, M. L. (2017a). Single or multiple frequency generators in on-going brain activity: a mechanistic whole-brain model of empirical MEG data. NeuroImage, 152, 538-550. doi:10.1016/j .neuroimage.2017.03.023. 
Deco, G., Kringelbach, M. L., Jirsa, V. K., \& Ritter, P. (2017b). The dynamics of resting fluctuations in the brain: metastability and its dynamical cortical core. Scientific Reports, 7, 1-14. doi:10.1038/s41598-017-03073-5.

Flandrin, P., Goncalves, P., \& Rilling, G. (2004). Detrending and denoising with empirical mode decompositions. In European Signal Processing Conference. volume 06-10-Sept.

Flandrin, P., Gonçalvès, P., \& Rilling, G. (2005). EMD equivalent filter banks, from interpretation to applications. (pp. 57-74). WORLD SCIENTIFIC volume 5 of Interdisciplinary Mathematical Sciences. URL: https://www.worldscientific.com/worldscibooks/10.1142/5862http: //www.worldscientific.com/doi/abs/10.1142/9789812703347\{_\}0003. doi:10.1142/9789812703347_0003.

Gensler, A., \& Sick, B. (2014). Novel criteria to measure performance of time series segmentation techniques. CEUR Workshop Proceedings, 1226, 193-204.

Granger, C. W., Newbold, P., \& Econom, J. (1974). Spurious regressions in econometrics. Baltagi, Badi H. A Companion of Theoretical Econometrics, 2, 557-61. URL: https://doi.org/10.1016/0304-4076(74)90034-7. doi:10.1016/0304-4076(74)90034-7.

Gustafsson, F. (1996). The marginalized likelihood ratio test for detecting abrupt changes. IEEE Transactions on Automatic Control, 41, 66-78. URL: http://ieeexplore.ieee.org/document/481608/. doi:10.1109/9.481608.

Gustafsson, F., \& Gustafsson, F. (2000). Adaptive filtering and change detection volume 1. Citeseer.

Hutchison, R. M., Womelsdorf, T., Allen, E. A., Bandettini, P. A., Calhoun, V. D., Corbetta, M., Della Penna, S., Duyn, J. H., Glover, G. H., Gonzalez-Castillo, J., Handwerker, D. A., Keilholz, S., Kiviniemi, V., Leopold, D. A., de Pasquale, F., Sporns, O., Walter, M., \& Chang, C. (2013). Dynamic functional connectivity: promise, issues, and interpretations. NeuroImage, 80, 360-378. URL: http://dx.doi.org/10.1016/j.neuroimage.2013.05.079. doi:10.1016/j. neuroimage. 2013.05.079. arXiv:NIHMS150003.

Idé, T. (2014). Change Detection from Heterogeneous Data Sources. In Data Mining for Service (pp. 221-243). volume 3. URL: http://link.springer.com/10.1007/978-3-642-45252-9http: //link.springer.com/10.1007/978-3-642-45252-9_13. doi:10.1007/978-3-642-45252-9_13.

Idé, T., Lozano, A. C., Abe, N., \& Liu, Y. (2009). Proximity-based anomaly detection using sparse structure learning. Society for Industrial and Applied Mathematics - 9th SIAM International Conference on Data Mining 2009, Proceedings in Applied Mathematics, 1, 96-107. doi:10.1137/1.9781611972795.9.

Kayser, J., Tenke, C. E., Gates, N. A., Kroppmann, C. J., Gil, R. B., \& Bruder, G. E. (2006). Erp/csd indices of impaired verbal working memory subprocesses in schizophrenia. Psychophysiology, 43, 237-252.

Ko, T.-W., \& Ermentrout, G. B. (2009). Phase-response curves of coupled oscillators. Physical Review E, 79, 016211. URL: https://link.aps.org/doi/10.1103/PhysRevE.79.016211. doi:10.1103/PhysRevE.79.016211. arXiv:arXiv:0809.3371v1.

Kovács, G., Sebestyen, G., \& Hangan, A. (2020). Evaluation metrics for anomaly detection algorithms in time-series. Acta Universitatis Sapientiae, Informatica, 11, 113-130. doi:10.2478/ausi-2019-0008.

Kuramoto, Y. (1984). Chemical oscillations, waves, and turbulence. Springer. URL: hhttps://www.springer.com/gp/book/9783642696916. doi:10.1007/978-3-642-69689-3.

Liu, S., Yamada, M., Collier, N., \& Sugiyama, M. (2013). Change-point detection in time-series data by relative density-ratio estimation. Neural Networks, 43, 72-83. doi:10.1016/j.neunet.2013.01.012. 
McFadden, K., Steinmetz, S., Carroll, A., Simon, S., Wallace, A., \& Rojas, D. (2013). EEG auditory steady state reliability paper, . URL: https:

//figshare.com/articles/dataset/EEG_Auditory_Steady_State_Reliability_Paper/829584. doi:10.6084/m9.figshare.829584.v14.

McFadden, K. L., Steinmetz, S. E., Carroll, A. M., Simon, S. T., Wallace, A., \& Rojas, D. C. (2014). Test-retest reliability of the $40 \mathrm{~Hz}$ EEG auditory steady-state response. PLoS ONE, 9, 59-61. doi:10.1371/journal.pone.0085748.

Molla, M. K. I., Tanaka, T., Rutkowski, T. M., \& Cichocki, A. (2010). Separation of EOG artifacts from EEG signals using bivariate EMD. In 2010 IEEE International Conference on Acoustics, Speech and Signal Processing (pp. 562-565). IEEE. URL: http://ieeexplore.ieee.org/document/5495594/. doi:10.1109/ICASSP. 2010.5495594.

Netoff, T., Schwemmer, M. A., \& Lewis, T. J. (2012). Phase response curves in neuroscience. URL: http://link. springer.com/10.1007/978-1-4614-0739-3. doi:10.1007/978-1-4614-0739-3.

Onojima, T., Goto, T., Mizuhara, H., \& Aoyagi, T. (2018). A dynamical systems approach for estimating phase interactions between rhythms of different frequencies from experimental data. PLoS Computational Biology, (pp. 1-20). doi:10.1371/journal.pcbi.1005928.

Ota, K., \& Aoyagi, T. (2014). Direct extraction of phase dynamics from fluctuating rhythmic data based on a bayesian approach. doi:10.1016/j.ceb.2004.02.009. arXiv:arXiv:1405.4126v1.

Penny, W. D., Litvak, V., Fuentemilla, L., Duzel, E., \& Friston, K. (2009). Dynamic Causal Models for phase coupling. Journal of Neuroscience Methods, 183, 19-30. URL: http://dx.doi.org/10.1016/j.jneumeth.2009.06.029. doi:10.1016/j.jneumeth.2009.06.029.

Pietras, B., \& Daffertshofer, A. (2019). Network dynamics of coupled oscillators and phase reduction techniques. Physics Reports, 819,1-105. URL: https://doi.org/10.1016/j.physrep.2019.06.001. doi:10.1016/j.physrep.2019.06.001.

Rauschecker, J. P., \& Scott, S. K. (2009). Maps and streams in the auditory cortex: nonhuman primates illuminate human speech processing. Nature Neuroscience, 12, 718-724. doi:10.1038/nn.2331.

Reyes, S. A., Salvi, R. J., Burkard, R. F., Coad, M. L., Wack, D. S., Galantowicz, P. J., \& Lockwood, A. H. (2004). PET imaging of the $40 \mathrm{~Hz}$ auditory steady state response. Hearing Research, 194, 73-80. doi:10.1016/j.heares. 2004.04.001.

Ross, B., Herdman, A. T., \& Pantev, C. (2005). Right hemispheric laterality of human $40 \mathrm{~Hz}$ auditory steady-state responses. Cerebral Cortex, 15, 2029-2039. doi:10.1093/cercor/bhi078.

Sarris, A. H. (1973). A bayesian approach to estimation of time-varying regression coefficients. Journal of economic and social measurement, 2, 501-523. URL: http://www.nber.org/chapters/c9941.

Sase, T., \& Kitajo, K. (2021). The metastable brain associated with autistic-like traits of typically developing individuals. PLOS Computational Biology, 17, e1008929. URL: http://dx.doi.org/10.1371/journal.pcbi.1008929. doi:10.1371/journal.pcbi.1008929.

Stankovski, T., Pereira, T., McClintock, P. V., \& Stefanovska, A. (2017). Coupling functions: universal insights into dynamical interaction mechanisms. Reviews of Modern Physics, 89, 045001. URL: https://link.aps.org/doi/10.1103/RevModPhys.89.045001. doi:10.1103/RevModPhys.89.045001. arXiv: 1706.01810.

Suzuki, K., Aoyagi, T., \& Kitano, K. (2018). Bayesian estimation of phase dynamics based on partially sampled spikes generated by realistic model neurons. Frontiers in Computational Neuroscience, 11, 1-13. URL: http://journal.frontiersin.org/article/10.3389/fncom.2017.00116/full. doi:10.3389/fncom.2017.00116. 
Tzikas, D. G., Likas, A. C., \& Galatsanos, N. P. (2008). The variational approximation for Bayesian inference: Life after the EM algorithm. IEEE Signal Processing Magazine, 25, 131-146.

doi:10.1109/MSP. 2008.929620.

Xiong, J., \& Zhou, T. (2013). A Kalman-Filter Based Approach to Identification of Time-Varying Gene Regulatory Networks. PLoS ONE, 8. doi:10.1371/journal.pone.0074571.

Yamanishi, K., \& Takeuchi, J. I. (2002). A unifying framework for detecting outliers and change points from non-stationary time series data. Proceedings of the ACM SIGKDD International Conference on Knowledge Discovery and Data Mining, (pp. 676-681). doi:10.1145/775107.775148.

Ying, J., Zhou, D., Lin, K., \& Gao, X. (2015). Network analysis of functional brain connectivity driven by gamma-band auditory steady-state response in auditory hallucinations. Journal of Medical and Biological Engineering, 35, 45-51. doi:10.1007/s40846-015-0004-0. 